\title{
Complex Brain Network Analysis and Its Applications to Brain Disorders: A Survey
}

\author{
Jin Liu, ${ }^{1}$ Min Li, ${ }^{1}$ Yi Pan, ${ }^{2}$ Wei Lan, ${ }^{1}$ Ruiqing Zheng, ${ }^{1}$ Fang-Xiang Wu, ${ }^{3}$ and Jianxin Wang ${ }^{1}$ \\ ${ }^{1}$ School of Information Science and Engineering, Central South University, Changsha 410083, China \\ ${ }^{2}$ Department of Computer Science, Georgia State University, Atlanta, GA 30302, USA \\ ${ }^{3}$ Division of Biomedical Engineering and Department of Mechanical Engineering, University of Saskatchewan, \\ Saskatoon, SK, Canada S7N5A9 \\ Correspondence should be addressed to Jianxin Wang; jxwang@mail.csu.edu.cn
}

Received 26 April 2017; Revised 18 September 2017; Accepted 27 September 2017; Published 22 October 2017

Academic Editor: Manlio De Domenico

Copyright (C) 2017 Jin Liu et al. This is an open access article distributed under the Creative Commons Attribution License, which permits unrestricted use, distribution, and reproduction in any medium, provided the original work is properly cited.

\begin{abstract}
It is well known that most brain disorders are complex diseases, such as Alzheimer's disease (AD) and schizophrenia (SCZ). In general, brain regions and their interactions can be modeled as complex brain network, which describe highly efficient information transmission in a brain. Therefore, complex brain network analysis plays an important role in the study of complex brain diseases. With the development of noninvasive neuroimaging and electrophysiological techniques, experimental data can be produced for constructing complex brain networks. In recent years, researchers have found that brain networks constructed by using neuroimaging data and electrophysiological data have many important topological properties, such as small-world property, modularity, and rich club. More importantly, many brain disorders have been found to be associated with the abnormal topological structures of brain networks. These findings provide not only a new perspective to explore the pathological mechanisms of brain disorders, but also guidance for early diagnosis and treatment of brain disorders. The purpose of this survey is to provide a comprehensive overview for complex brain network analysis and its applications to brain disorders.
\end{abstract}

\section{Introduction}

The consensus of the neuroscience community is that a human brain contains about 100 billion $\left(10^{11}\right)$ neurons connected by about 100 trillion $\left(10^{14}\right)$ synapses $[1,2]$, which are anatomically organized over multiple space scales and functionally interact over multiple time scales. Therefore, exploring the brain and revealing the neural mechanism of brain activities have been a challenging scientific problem [35]. Now it is realized that brain functions are determined not only by a single neuron or a single brain region independently, but also by clusters of neurons, neural circuits within a function block, or a group of interactions between brain regions [6]. A brain can be modeled as a complex network [79], which enables highly efficient information transmission. Currently, network neuroscience has become a research hotspot [10-12].

Clinical disorders of human brain networks, such as Alzheimer's disease (AD), schizophrenia (SCZ), and
Parkinson's disease (PD), are among the most disabling and therapeutically intractable health problems. Therefore, it is unsurprising that understanding brain network connectivity has long been a central goal of neuroscience and has recently catalyzed an unprecedented era of large-scale initiatives and collaborative projects, such as BRAIN Initiative (http://www.braininitiative.org/) (USA, 2013), Human Brain Project (https://www.humanbrainproject.eu/) (Europe, 2013), Brain/MINDS Project (http://brainminds.jp/) (Japan, 2014), Australian Brain Alliance (http://www.cibf.edu.au/australian-brain-alliance) (Australia, 2016), and China Brain Project (China, 2016) [13]. The goal of these projects is to revolutionize our understanding of the human brain. By accelerating the development and application of innovative technologies, revolutionary new accurate images of the brain can be produced for more accurate understanding of brain functions.

Neuroimaging techniques provide a way for clinicians and researchers to examine the structural and functional changes in the brain disorders in vivo [14-26]. Commonly 


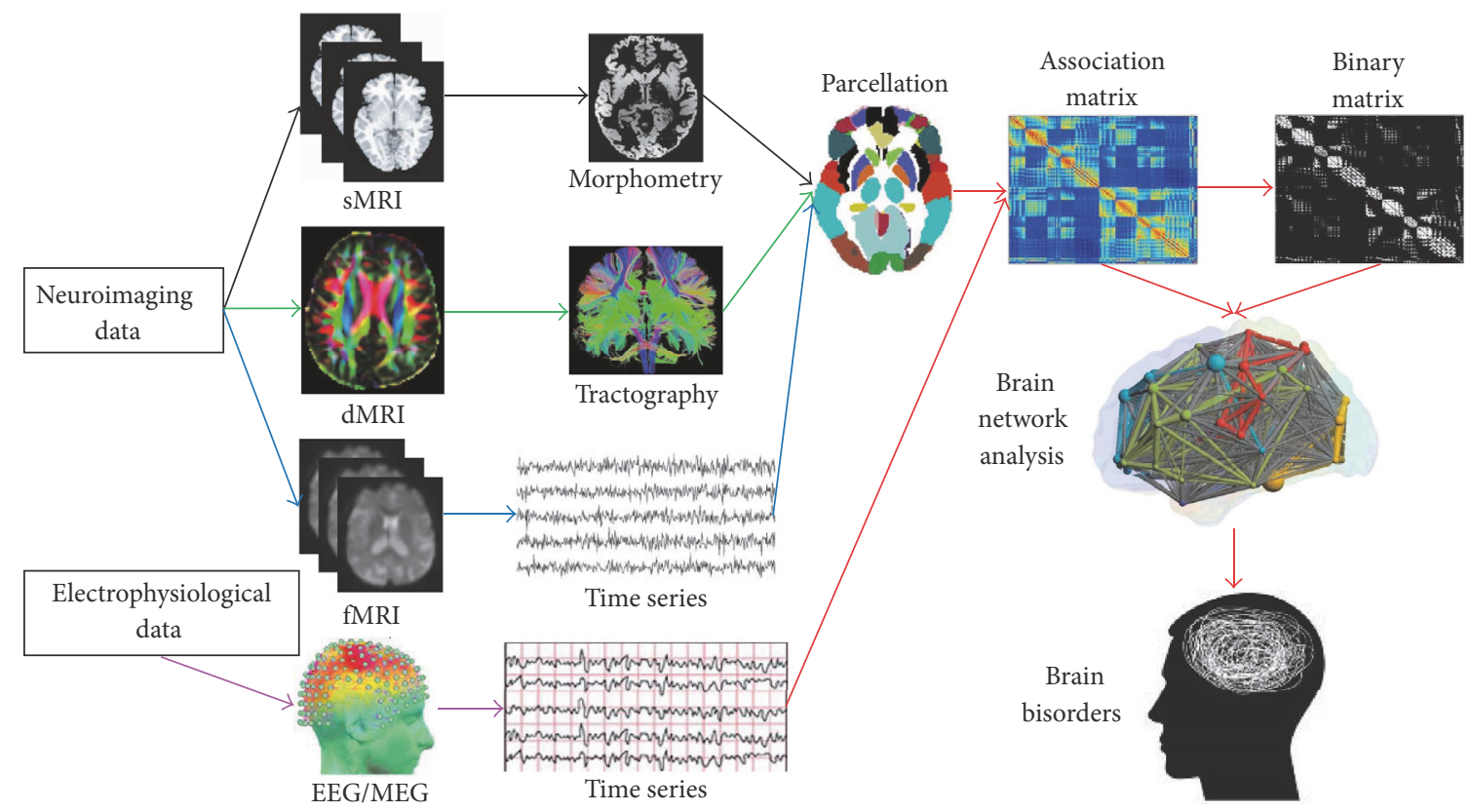

FIGURE 1: A general perspective for complex brain network analysis methods and its applications in brain disorders.

used modalities include structural magnetic resonance imaging (sMRI, such as T1w MRI) [27], diffusion magnetic resonance imaging (dMRI, such as diffusion tensor imaging (DTI)) [28], and functional magnetic resonance imaging (fMRI, such as rs-fMRI) [29, 30]. Electroencephalography (EEG) [31] and magnetoencephalography (MEG) [32] are noninvasive electrophysiological techniques for recording brain activities. EEG is used to measure voltage sensed by an array of electrodes placed on the scalp. MEG is used to measure the magnetic field outside the head using an array of very sensitive magnetic field detectors (magnetometers). The signals recorded by EEG and MEG directly reflect current flows generated by neurons within a brain. EEG/MEG also have been utilized for the studies of brain disorders [3336]. Network-based analysis has been widely used in various fields, such as medical image analysis [37-40] and bioinformatics [41-44]. The two techniques (i.e., neuroimaging and magnetoencephalography) have been used to construct brain networks with multiple different scales, which have led to the development of brain network studies [45-48]. The construction of brain networks provides a necessary basis for brain network analysis, which includes global efficiency, local efficiency, modularity, and rich club $[49,50]$. A graph can effectively and visually present a brain as a complex network whose topological structures can be quantified [5153]. Therefore, graph theory has become one of the most important mathematical tools in the field of brain network analysis [54-56].

In this article, we provide a comprehensive review regarding complex brain network analysis and its applications to brain disorders as shown in Figure 1. Firstly, we introduce some basic concepts for constructing the brain networks based on neuroimaging and electrophysiological data, which include structural data and functional data. It is worth mentioning that, in this article, structural data only consider sMRI and dMRI and functional data only consider fMRI and EEG/MEG. After summarizing methods for brain network analysis based on graph theory, we present several applications of brain network analysis in brain disorders. Finally some conclusions are drawn and the directions of future work are pointed out along with brain network analysis.

\section{Brain Network Construction}

A brain network is typically represented by a graph $G=$ $(V, E)$, where $V$ is the set of vertices (or nodes) and $E$ is the set of edges (or links, also called connections) between pairs of nodes. As nodes and edges are the basic elements of each brain network, the accurate definition of the two elements plays important roles in the brain network analysis [57].

2.1. Nodes. In order to construct a brain network, the first step is to define nodes of the brain network. The nodes should represent different, functionally uniform neurons (which are grouped together to perform the same function) or brain regions. However, since there is no gold standard for brain parcellation, methods for defining nodes of brain networks are varied as follows:

(i) The simplest method is to treat each measurement point as a separate node. This method occurs before the data acquisition. For example, different nodes could correspond to separate voxels in MRI images, different sensors in MEG, or different electrodes in EEG. The advantage of this method is that no additional data processing or assumptions are required to analyze the data at the original resolution or to perform further averaging or aggregating. The weaknesses of this method include the following: (1) 
TABLE 1: Some common atlas in brain network study.

\begin{tabular}{lcccc}
\hline Name & Number of regions & Links & Reference \\
\hline Brodmann area & 104 & http://www.fmriconsulting.com/brodmann/Interact.html & [58] \\
Anatomical Automatic Labeling atlas & 116 & http://www.cyceron.fr/index.php/fr/plateforme/freeware & [59] \\
Destrieux atlas & 148 & https://surfer.nmr.mgh.harvard.edu/fswiki/CorticalParcellation & [90, 91] \\
Desikan-Killiany atlas & 68 & https://surfer.nmr.mgh.harvard.edu/fswiki/CorticalParcellation & [92] \\
LPBA40 & 56 & http://neuro.imm.dtu.dk/wiki/LPBA40 & [93] \\
Brainnetome atlas & 246 & http://atlas.brainnetome.org/ & [94] \\
HCP MMP1.0 & 360 & https://balsa.wustl.edu/study/show/RVVG & [69] \\
\hline
\end{tabular}

there is no guarantee that the measurement points are consistent with the boundaries of functional human cell populations; (2) the boundaries of a specific, functionally specialized human cell population may go beyond the boundaries of a voxel. Thus, this method is often used in EEG/MEG but is rarely used in the other three types of data (i.e., sMRI, dMRI, and fMRI).

(ii) The most common method is to register experimental data to an a priori anatomical parcellation atlas, such as Brodmann area [58] and Anatomical Automatic Labeling (AAL) atlas [59]. For more atlases, please see Table 1 . The advantage of this method is that it can easily parcellate a whole brain into many regions (about $10^{2}$ ) as nodes of a brain network. The weakness of this method is that the resulting regions can show considerable variation in size, which affects any subsequent brain network analysis.

(iii) Based on the problem of the size of the regions, the alternative method is to treat each voxel as a separate node. The only difference from the first method is that this method occurs after the data acquisition. The advantage of this method is that it can construct a very large, high-resolution brain network (more than $10^{4}$ nodes) for each brain. The weaknesses of this method include the following: (1) it could yield noise and thus affect the subsequent brain network analysis; (2) as the resulting brain network is large, it can cause difficulties in brain network analysis, such as looking for modularity.

(iv) The fourth method is to define nodes according to some a priori criteria. For example, some researchers have mapped activation patterns in a specific task and defined activation regions as the nodes of brain networks of interest according to these mappings [60]. Meanwhile, some researchers have applied metaanalysis methods to identify important brain regions as the nodes of brain networks of interest [61]. The advantage of this method is that the determination of the nodes is based on the measurement of brain functions, which can be adjusted according to specific hypotheses about brain networks of interest. The weakness of this method is that the resulting nodes may not be used in different modalities. For example, in fMRI, the nodes can be defined by a specific task and are usually included within the gray matter. However, in dMRI, the resulting nodes may make it difficult to track connections, since most fiber tracking methods are difficult to reconstruct the pathways of the axons within gray matter [62].

(v) The fifth method uses connectivity to define nodes. The essence of this method is to measure the connectivity of each voxel to all other voxels, and then some voxels are clustered together as brain regions with a specific function if these voxels have a similar connectivity [63]. For example, Anwander et al. [64] used an automatic clustering method to identify cortical regions with internally coherent connectivity in DTI and parcellate Broca's area to three subregions, which include BA44, BA45, and the deep frontal operculum. The advantage of this methods is that it can find brain regions with specific functions as the nodes of brain networks of interest. The weakness of this method is that since spatially separating brain regions may have similar connectivity, there is no guarantee that the resulting nodes are composed of a number of voxels that are spatially continuous [65, 66]. To address the weakness of this method, some methods with spatial constraints have been proposed $[67,68]$.

(vi) The sixth method is to define nodes by combining pieces of multimodal information, such as anatomical homogeneity [69] and synchrony [65]. The advantage of this method is that it can obtain complementary information from the multimodal data so that the location of the nodes is more accurate. The weaknesses of this method include the following: (1) it may have more noise than other methods with single modality data and thus affect the location of the nodes; (2) since it uses multimodal information, the cost of computation is also very large.

Based on the discussion about the above five methods, the definition of nodes is still a very challenging problem in order to obtain accurate results from brain network analysis.

2.2. Edges. The edges of a brain network represent the connectivity between two brain regions. Brain network connectivity can be divided into three types: structural connectivity, functional connectivity, and effective connectivity $[39,51,70]$. Structural connectivity contains two types: (1) the anatomical 
connections between neural elements, such as fiber bundles; (2) the interregional covariation of specific morphometric parameters, such as gray matter thickness. Functional connectivity refers to a statistical dependence between neural elements with physiological recordings or neurophysiological signals. The purpose of effective connectivity is to uncover the direct, causal influences that neural elements exert over each other's activity. Since there are relatively few studies of effective connectivity in brain networks, in this article we mainly consider the studies of structural connectivity and functional connectivity.

2.2.1. Edges Based on Structural Connectivity. In brain network study, there are two common types of neuroimaging techniques that are often used to define structural connectivity. The two types of neuroimaging techniques are sMRI and dMRI as shown in Figure 1. How to quantify structural connectivity based on sMRI and dMRI is described as follows:

(i) In sMRI, structural connectivity is indirectly estimated by calculating interregional correlation of specific morphometric parameters, such as gray matter volume or cortical thickness [71-73]. In this method, a measure (such as gray matter volume, cortical thickness, or other similar metrics) of each brain region is extracted, and then correlations between two brain regions are calculated as the edges of the brain network of interest. Morphological measurements of brain regions can be implemented by some open source tools such as FreeSurfer (http://www.freesurfer.net/) and SPM (http:// www.fil.ion.ucl.ac.uk/spm/). Pearson correlation and partial correlation are two of the most common methods to compute structure connectivity from sMRI images.

(ii) The most common technique for studying structural connectivity is dMRI [74-77]. In dMRI, the trajectories (connectivity) of axonal fibers can be reconstructed by using tractography, which includes deterministic tractography $[78,79]$ and probabilistic tractography [80].

(a) The deterministic tractography is simple and effective, and the satisfactory reconstruction can be obtained in some brain disorders. However, the weakness of this tractography is that an initial seed (i.e., a specific voxel) does not change during the reconstruction process. If the seed changes, the reconstruction is likely to produce a deviation. This tractography can be implemented by some common open source tools such as Diffusion Toolkit and Trackvis (http://trackvis.org/), DTIStudio (https://www .dtistudio.org/), and medInria (http://med.inria .fr/).

(b) The advantage of the probabilistic tractography is that the reconstruction results are more stable to noise, and fiber cross problem can be improved to some extent. The weakness of this method is computationally intensive and time consuming. This tractography can be also implemented by some common open source tools such as FSL (https://fsl.fmrib.ox.ac.uk/ fsl/fslwiki), MRItrix3 (http://www.mrtrix.org/), and DSIStudio (http://dsi-studio.labsolver.org/).

For more details about the above two tractographies, please see [81, 82]. After this step, structural connectivity can be estimated by several different measures of connectivity strength. The simplest measure is that the number of axonal fibers (FN) connecting two brain regions is used as connectivity strength, which is the weight of the edge of the brain network of interest. Another common measure of connectivity strength is the average fractional anisotropy (FA) value of all voxels over the reconstructed tract between two brain regions.

Based on the above analysis, in both sMRI and dMRI, the resulting edges are undirected and weighted. Thus, the resulting connectivity matrix of each brain is symmetric and generates a weighted undirected network. In some studies, the weighted undirected resulting networks were converted into binary undirected networks by a specific threshold $[72$, $83,84]$.

2.2.2. Edges Based on Functional Connectivity. In brain network study, functional connectivity is often defined by using fMRI, EEG, and MEG as shown in Figure 1. As can be seen from Figure 1, neurophysiological signals (such as time series) can be extracted from fMRI and EEG/MEG. EEG/MEG offer high temporal resolution, which allows brain activity to be sampled on the millisecond ranges that match with the speed of neural signals. fMRI generally offers a higher spatial resolution than EEG/MEG, but its temporal resolution is relatively low.

Functional connectivity reflects the statistical correlation between neurophysiological signals (such as time series) recorded from each brain region. The correlation can be measured by using various methods. The main measurement methods are divided into two categories: linear methods and nonlinear methods. The common linear methods $[85,86]$ mainly include Pearson correlation, partial correlation, and partial coherence. The common nonlinear methods mainly include synchronization likelihood [21,34], mutual information [87], and wavelet correlation [88, 89]. For example, the Pearson correlation between brain regional activity time series is calculated as the edges of the brain network of interest, which are weighted and undirected. Thus, the resulting brain networks are also weighted undirected networks. Similarly, such weighted undirected resulting networks can also be converted into binary undirected networks by a specific threshold $[33,85,87]$.

\section{Brain Network Analysis}

In general, the topology of networks can be divided into four types: binary undirected, binary directed, weighted 


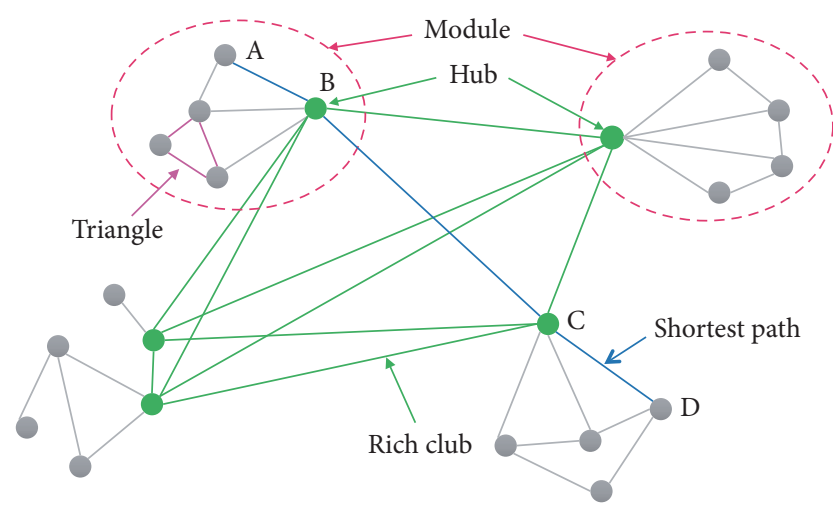

FIgURE 2: An example of a simple binary undirected network (graph).

undirected, and weighted directed. In this article, since we only focus on structural connectivity and functional connectivity, only two types of brain networks are taken into consideration: weighted undirected and binary undirected.

\subsection{Degree, Clustering Coefficient, and Shortest Path Length.} Node degree is one of the most elementary and important measures for a brain network and is often denoted as $k$. The degree of a node is the number of edges connecting the node with all other nodes. In general, the greater the degree of a node is, the more the nodes it connected to are and the more important it may be in the brain network. In a binary undirected network, the degree $k_{i}$ of a node $i$ is defined as

$$
k_{i}=\sum_{j \neq i} a_{i j}
$$

where $a_{i j}=1$ if the connection of node $i$ and node $j$ exists; otherwise $a_{i j}=0$. For example, the degree of node $\mathrm{C}$ in Figure 2 is 7 . The strength of a network is the average of the degree across all of the nodes in the network. Thus, for a binary undirected brain network, the network strength $S$ can be calculated by

$$
S=\frac{1}{N} \sum_{i \in N} k_{i}
$$

Degree distribution $P(k)$ is also a basic topological characterization and is defined as the fraction of nodes with degree $k$ in the whole brain network in practical application. For example, if there are totally $N$ nodes in a brain network where there are $N_{k}$ nodes with degree of $k$,

$$
P(k)=\frac{N_{k}}{N} .
$$

For many brain networks, the degree distribution is characterized by a fat tail that indicates the presence of central position nodes. These central position nodes usually play a vital role in the convergence and divergence of information in the brain network [138]. In the field of brain networks, if a node occupies a central position in the overall organization of a brain network, the node can be called hub node [139]. For example, the green nodes (such as nodes $\mathrm{B}$ and $\mathrm{C}$ ) in Figure 2 are considered as hubs in the simple binary undirected network.

A subgraph with 3 nodes and 3 edges is called a triangle as shown in Figure 2 (pink). In a network, the number of triangles $t_{i}$ around a node $i$ is defined as

$$
t_{i}=\frac{1}{2} \sum_{i \leftrightarrow j \leftrightarrow h} a_{i j} a_{i h} a_{j h} .
$$

The local clustering coefficient of a node measures the possibility that any two neighbors of the node are also connected. In this article, for convenience, the local clustering coefficient is described by clustering coefficient. In a binary undirected network, the clustering coefficient $C(i)$ of a node $i$ is equal to the ratio of the number of the actual connected edges between its adjacent nodes to the number of all possible connection edges; that is,

$$
C(i)=\frac{2 t_{i}}{k_{i}\left(k_{i}-1\right)} .
$$

The average clustering coefficient of all nodes in a network is defined as the clustering coefficient of the network:

$$
C=\frac{1}{N} \sum_{i} C(i)=\frac{1}{N} \sum_{i} \frac{2 t_{i}}{k_{i}\left(k_{i}-1\right)} .
$$

The clustering coefficient is a measure of functional segregation, which is the ability for specialized processing to occur within densely interconnected groups of brain regions [140].

The shortest path plays an important role in the information transmission of a brain network, and it is a very important measure to describe the internal structure of the brain network. The shortest path can transmit the information more quickly and reduce brain consumption. In a binary undirected network, a path between nodes $i$ and $j$ with the minimum number of edges is called the shortest path between these two nodes and its length $l_{i j}$ is denoted as

$$
l_{i j}=\sum_{a_{s t} \in l_{i \leftrightarrow j}} a_{s t},
$$

where $l_{i \rightarrow j}$ is the shortest path between nodes $i$ and $j$. For example, the shortest path length of nodes $\mathrm{A}$ and $\mathrm{D}$ in Figure 2 is 3 (i.e., $l_{\mathrm{AD}}=3$ ). The average shortest path length between node $i$ and other all nodes is denoted as $l_{i}$ :

$$
l_{i}=\frac{1}{(N-1)} \sum_{i \neq j} l_{i j} .
$$

The characteristic path length $L$ of a network is the average shortest path length between all possible pairs of nodes in the network and is defined as

$$
L=\frac{1}{N} \sum_{i} l_{i} .
$$

The characteristic path length is a measure of functional integration, which is the ability to rapidly combine pieces of specialized information from distributed brain regions [140]. 
3.2. Centrality. The centrality is to measure the importance of nodes in a brain network. The higher the centrality of a node is, the more effective the node is in the information transmission of the brain network. In the brain network analysis, three measures of centrality are often used, including degree centrality, closeness centrality, and betweenness centrality as follows:

(i) Degree centrality is the most common measure of centrality, which uses the degree of a node to describe the importance of the node in the brain network. In brain network analysis, the degree centrality of a brain region measures the direct impact of the brain region on other adjacent brain regions. Thus, in a binary undirected network, the degree centrality $C_{d}(i)$ of a node $i$ is equivalent to the degree of the node:

$$
C_{d}(i)=k_{i}=\sum_{j \neq i} a_{i j}
$$

(ii) Closeness centrality [141] reflects the closeness between a node and other nodes in a brain network. Thus, the closer a node is to all other nodes in the brain network, the higher the centrality of the node is. In brain network analysis, the closeness centrality of a brain region measures the indirect impact of the brain region on other brain regions. For a binary undirected network, the closeness centrality $C_{c}(i)$ of a node $i$ is defined as the inverse of the average shortest path length of the node to all other nodes:

$$
C_{c}(i)=\frac{N-1}{\sum_{j \neq i} l_{i j}} .
$$

(iii) Betweenness centrality $[142,143]$ is a very popular measure, which quantifies the number of times that a node acts as a bridge along the shortest path between two other nodes. In brain network analysis, the betweenness centrality of a brain region measures the impact of the brain region on the flow of information across the brain network. In a binary undirected network, the betweenness centrality $C_{b}(i)$ of a node $i$ is defined as the proportion of shortest paths between nodes $j$ and $h$ that pass through $i$ :

$$
C_{b}(i)=\frac{2}{(N-1)(N-2)} \sum_{j \neq h \neq i} \frac{n_{h j}(i)}{n_{h j}},
$$

where $n_{h j}(i)$ is the number of shortest paths between $h$ and $j$ that pass through $i, n_{h j}$ is the number of all shortest paths between $h$ and $j$, and $(N-1)(N-2) / 2$ is the number of node pairs that do not include node $i$.

In brain network analysis, the above three centrality measures are also often used to identify hub brain regions. Firstly, the centrality values of all brain regions are ranked. Then, a specific threshold (e.g., mean + square deviation) is used to determine the hub nodes. That is, the brain regions whose centrality values are larger than the specific threshold are considered as hubs. For example, node B in Figure 2 is a hub of the simple network in terms of degree centrality.
3.3. Efficiency. The efficiency of a network (such as brain network) measures the ability of the network to exchange information. The higher the efficiency of the network is, the stronger the ability of information exchange is. The efficiency of a network mainly considers global efficiency and local efficiency. The global efficiency measures the ability of parallel information exchange across the whole network, while the local efficiency measures the ability of fault tolerance of a network [144].

Both global efficiency and local efficiency are closely related to nodal efficiency. Nodal efficiency measures how well a specific region is integrated within the network via its shortest paths. In a binary undirected network, the efficiency $E_{\text {nodal }}(i)$ of a node $i$ is defined as the normalized sum of the reciprocal of the shortest path lengths from the node to all other nodes of the network:

$$
E_{\text {nodal }}(i)=\frac{1}{N-1} \sum_{i \neq j} \frac{1}{l_{i j}} .
$$

Obviously, the shorter the shortest path lengths of a node is, the higher the efficiency of the node is. The nodes with high nodal efficiency play an important role in the information integration and distribution [139]. It is worth mentioning that the nodes with high nodal efficiency can also be seen as hubs [130].

The global efficiency of a network [145] is the average nodal efficiencies of all nodes in the network and is defined as

$$
E_{\text {glob }}=\frac{1}{N} \sum_{i} E_{\text {nodal }}(i)=\frac{1}{N(N-1)} \sum_{i \neq j} \frac{1}{l_{i j}} .
$$

Since the brain is considered as a multiactivity parallel system, it should have a high global efficiency in a brain network [144].

The local efficiency of a node can be regarded as the global efficiency of the subnetwork containing itself and its all direct neighbors. In a binary undirected network, the local efficiency $E_{\text {loc }}(i)$ of a node $i$ can be defined as

$$
E_{\mathrm{loc}}(i)=\frac{1}{N_{G_{i}}\left(N_{G_{i}}-1\right)} \sum_{j \neq h \in G_{i}} \frac{1}{l_{j h}},
$$

where $G_{i}$ is the subgraph that consists of node $i$ and its all direct neighbors.

Similarly, the local efficiency of a network is the average local efficiencies of all nodes in the network and is computed by

$$
E_{\mathrm{loc}}=\frac{1}{N} \sum_{i} E_{\mathrm{loc}}(i) .
$$

It is worth mentioning that the difference between nodal efficiency and local efficiency of a node is that the former measures the ability of information exchange of the node itself, while the latter measures the ability of information exchange of the subnetwork consisting of itself and its all direct neighbors. 
3.4. Modularity and Rich Club. A module is a group of nodes with dense internal connections but sparse external connections in a network as shown in Figure 2. The real network often has a number of relatively independent and interrelated modules that have different functions and evolve independently without affecting other modules. At the same time, the modular structure also provides more detailed roles and properties of nodes. For example, some nodes are important in their modules but are not necessarily important for the entire network; these nodes are called provincial hubs; while some other nodes though in their own modules are limited, they are connected to different modules, maintaining the connectivity of the entire network. Therefore these nodes play an important role in information transmission throughout the network and are called connector hubs. A participation index [146] is used to determine whether a node is a provincial hub or a connector hub. The participation index $\left(P_{i}\right)$ of node $i$ is computed by

$$
P_{i}=1-\sum_{m=1}^{N_{m}}\left(\frac{k_{i m}}{k_{i}}\right)^{2}
$$

where $N_{m}$ is the number of modules and $k_{i m}$ is the number of connections from node $i$ to module $m$. In general, if $P_{i}$ is greater than a specific threshold, node $i$ is a connector hub, and otherwise it is a provincial hub.

Biological networks, including human brains, exhibit a high degree of modularity. In complex network analysis, modularity is used to measure the quality of division of a network into modules [147]. Currently, there are different methods to calculate the modularity of a brain network [148]. Here, we introduce two common modularity measures in brain network analysis. Given that a brain network is fully subdivided into several nonoverlapping modules $M$, these two common modularity measures are computed as follows:

(i)

$$
Q=\sum_{s \in M}\left[p_{s s}-\left(\sum_{t \in M} p_{s t}\right)\right],
$$

where $p_{s s}$ is the proportion of existing links in module $s$ and $p_{s t}$ is the proportion of existing links between modules $s$ and $t$.

(ii)

$$
Q=\frac{1}{2 N(N-1)} \sum_{i j}\left(a_{i j}-\frac{k_{i} k_{j}}{N(N-1)}\right) \delta\left(m_{i}, m_{j}\right),
$$

where $m_{i}$ and $m_{j}$ are the modules containing node $i$ and node $j$, respectively, and if nodes $i$ and $j$ are in the same module, $\delta\left(m_{i}, m_{j}\right)=1$; otherwise, $\delta\left(m_{i}, m_{j}\right)=$ 0 .

The so-called "rich club" effect in complex networks is that the hubs of a complex network tend to be more densely connected among themselves than nodes of a lower degree [149-151] as shown in Figure 2. In essence, nodes with a large number of edges, which are usually called rich nodes, are much more likely to form closely interconnected subgraphs (also called clubs) than low-degree nodes. The normalized rich club coefficient $\left(\rho_{\text {rand }}(k)\right)$ is used to quantify the rich club effect of a complex network $[149,150]$ and is defined as

$$
\begin{aligned}
\rho_{\text {rand }}(k) & =\frac{\phi(k)}{\phi_{\text {rand }}(k)} \\
\phi(k) & =\frac{2 E_{>k}}{N_{>k}\left(N_{>k}-1\right)},
\end{aligned}
$$

where $N_{>k}$ is the number of nodes with degree larger than $k, E_{>k}$ is the number of edges connecting these $N_{>k}$ nodes, $N_{>k}\left(N_{>k}-1\right) / 2$ is the maximum possible number of edges among these nodes $N_{>k}, \phi(k)$ is the rich club coefficient of a given degree $k$, and $\phi_{\text {rand }}(k)$ is the rich club coefficient on a maximally randomized network with the same degree distribution of the network under study.

The rich club effect of brain networks plays an important role in global brain information transmission [152], which provides important information on the higher-level topology of brain networks. The rich club serves as an important backbone for a number of coactivation patterns among brain regions [153]. Thus, a rich club in a brain network is also crucial for promoting and integrating various segregated functions [148]. In brain network, a normalized rich club coefficient increasing with the degree $k$ indicates the dominance of a number of highly connected and mutually communicating brain regions, as opposed to a set consisting of many loosely connected and relatively independent brain regions.

3.5. Small-World Network. The concept of small-world networks was first proposed by Watts and Strogatz [154]. The small-world networks have both high clustering characteristics similar to regular networks and shorter shortest path lengths similar to random networks. In other words, The small-world networks combine the respective topological advantages of both regular networks and random networks to ensure the efficiency of information transmission at both local and global levels.

To determine whether a network is a small-world network, the following three criteria are employed:

$$
\begin{aligned}
& \gamma=\frac{C}{C_{\text {rand }}} \\
& \lambda=\frac{L}{L_{\text {rand }}} \\
& \sigma=\frac{\gamma}{\lambda},
\end{aligned}
$$

where $C_{\text {rand }}$ and $L_{\text {rand }}$ are the average clustering coefficient and characteristic path length of $M$ matched random networks that preserve the same number of nodes, edges, and degree distribution as the real network, $\gamma$ and $\lambda$ are the normalized clustering coefficient and normalized characteristic path length of the network, and $\sigma$ is the small-world index 
of the network [155]. A network with small-world property needs to meet two conditions: $\gamma \gg 1$ and $\lambda \approx 1$. Thus, the small-world index $\sigma>1$.

The brain supports both segregated and integrated information processing. Small-world topology comprises both high clustering (compatible with segregated processing) and short path length (compatible with integrated processing). Therefore, for a given brain network, if the brain network is found to have small-world property, it shows that the brain has better information processing performance, at least without great disruption.

In addition to the above important topological properties, there are many other properties in graph theory to characterize brain networks, such as hierarchy, motif, assortativity, and transitivity. For more details about brain network analysis, please see $[140,148,156]$. The brain network analysis can be also implemented by some common open source tools such as Brain Connectivity Toolbox (http://www.nitrc .org/projects/bct/), GRETNA (https://www.nitrc.org/projects/gretna/), and BrainWave (http://home.kpn.nl/stam7883/ brainwave.html).

\section{Applications of Brain Network Analysis}

Brain network analysis becomes increasingly important when either studying pathophysiology or exploring the networkbased biomarkers of brain disorders. The studies of brain network analysis with graph theoretical frameworks have been extensively applied to identify detailed abnormalities of network topologies associated with various brain disorders, including AD, SCZ, PD, and multiple sclerosis. In this section, we summarize the recent progress of structural and functional brain networks with several brain disorders, focusing on the changes in the topological organization of structural and functional brain networks in terms of graph theoretical frameworks.

4.1. Alzheimer's Disease. Alzheimer's disease (AD) is a degenerative brain disease and the most common type of dementia, comprising $60-80 \%$ of all dementia cases. In the US, there are more than 5.2 million AD patients in 2014, and it is estimated that 13.8 million Americans are AD patients by 2050 [157]. Thus, early diagnosis and treatment of $\mathrm{AD}$, especially at its prodromal stage such as MCI [158], have become a crucial step to delay or even avoid dementia. The clinical symptoms of $\mathrm{AD}$ are impairments of memory, language, and other cognitive functions, which seriously affect the daily life of patients and their families. Existing studies have demonstrated that these impairments are associated with abnormal structural and functional brain networks $[83,95,103,159]$.

4.1.1. Structural Brain Networks in AD. Structural brain networks of human beings can be constructed by using sMRI and dMRI. In this section, we review recent progress in analyzing the networks based on sMRI and dMRI in AD as shown in Table 2.

(i) Using sMRI: He et al. [95] first used cortical thickness measurement to investigate structural brain networks of $92 \mathrm{AD}$ patients and 97 healthy controls (HCs). They found increased clustering coefficient and shortest paths in $\mathrm{AD}$, implying an abnormal small-world property. In addition, they found reduced betweenness centrality in the temporal and parietal heteromodal association cortex regions and increased betweenness centrality in the occipital cortex regions. Yao et al. [83] used gray matter volumes to construct structural brain networks of $91 \mathrm{AD}$ patients, 113 MCI patients, and 98 HCs. Among structural brain networks of three groups, they found the greatest clustering coefficient and the longest absolute path length in AD. Their finding was similar to that by $\mathrm{He}$ et al. [95]. In addition, they found the smallworld index of the MCI networks was between $\mathrm{AD}$ and HC networks. Their finding showed that MCI is a transitional stage between $\mathrm{HC}$ and $\mathrm{AD}$. Compared with the $\mathrm{HCs}$, the MCI and $\mathrm{AD}$ patients retained hub regions in the frontal lobe but lost hub regions in the temporal lobe. Tijms et al. [96] investigated the topology properties of single-subject gray matter networks in $\mathrm{AD}$ and found decreased normalized clustering coefficient and normalized path length. Their finding is contrary to the previous two findings. Moreover, they found decreased small-world index in AD. Pereira et al. [97] constructed structural brain networks of stable MCI (sMCI) patients, late MCI converters (lMCIc), early MCI converters (eMCIc), and $\mathrm{AD}$ patients to investigate topology structure across groups. They found that, compared with the HC group, all patient groups exhibited increased path length, reduced transitivity, and increased modularity, and the patient group showed decreased smallworld index. In addition, compared with the sMCI group, other three patient groups showed decreased path length and clustering coefficient.

(ii) Using dMRI: Lo et al. [98] used DTI to construct structural brain networks of $25 \mathrm{AD}$ patients and 30 HCs. They found that although the two groups had a small-world property, the AD group showed increased shortest path length compared with the HC group. In addition, they found decreased global efficiency and reduced nodal efficiency in the frontal regions in AD. Bai et al. [99] considered two high risk groups, remitted geriatric depression (RGD) and amnestic MCI (aMCI), and constructed structural brain networks of the two groups using DTI and deterministic tractography. They found reduced network strength, reduced global efficiency, and increased absolute path length for both the RGD and aMCI patients compared with HCs, and there were no significant differences in these global network properties between the two high risk groups. Compared with HCs, they found that the two high risk groups had similar deficits of the regional and connectivity characteristics in the frontal regions. From comparison of RGD and aMCI, they found that the nodal efficiency of networks in the two groups was different in the posterior cingulate cortex 
TABLE 2: Overview of structural brain network studies in AD.

\begin{tabular}{|c|c|c|c|c|c|}
\hline Study & Modality & Subjects & Node definition & $\begin{array}{c}\text { Edge } \\
\text { definition }\end{array}$ & Network type \\
\hline $\begin{array}{l}\text { He et al., } 2008 \\
{[95]}\end{array}$ & sMRI & $\begin{array}{l}92 \mathrm{AD} \\
97 \mathrm{HC}\end{array}$ & $\begin{array}{l}54 \text { regions in } \\
\text { ANIMAL package }\end{array}$ & $\begin{array}{l}\text { Partial } \\
\text { correlation } \\
\text { based on } \\
\text { cortical } \\
\text { thickness }\end{array}$ & Binary \\
\hline $\begin{array}{l}\text { Yao et al., } 2010 \\
{[83]}\end{array}$ & sMRI & $\begin{array}{c}91 \mathrm{AD} \\
113 \mathrm{MCI} \\
98 \mathrm{HC}\end{array}$ & $\begin{array}{c}90 \text { regions in } \mathrm{AAL} \\
\text { atlas }\end{array}$ & $\begin{array}{l}\text { Pearson } \\
\text { correlation } \\
\text { based on } \\
\text { gray matter } \\
\text { volume }\end{array}$ & Binary \\
\hline $\begin{array}{l}\text { Tijms et al., } \\
2013 \text { [96] }\end{array}$ & sMRI & $\begin{array}{l}38 \mathrm{AD} \\
38 \mathrm{HC}\end{array}$ & $8683 \pm 545$ cubes & $\begin{array}{c}\text { Intracortical } \\
\text { similarity }\end{array}$ & Binary \\
\hline
\end{tabular}


TABLE 2: Continued.

\begin{tabular}{|c|c|c|c|c|c|c|}
\hline Study & Modality & Subjects & Node definition & $\begin{array}{c}\text { Edge } \\
\text { definition }\end{array}$ & Network type & Main findings \\
\hline $\begin{array}{l}\text { Wang et al., } \\
2016 \text { [101] }\end{array}$ & DTI & $\begin{array}{l}26 \mathrm{AD} \\
16 \mathrm{HC}\end{array}$ & $\begin{array}{c}90 \text { regions in AAL } \\
\text { atlas }\end{array}$ & FN & Binary & $\begin{array}{l}\text { (1) Higher small-world index in AD. } \\
\text { (2) Decreased global efficiency and } \\
\text { local efficiency in AD. } \\
\text { (3) Increased normalized shortest path } \\
\text { length and normalized clustering } \\
\text { coefficient in AD. }\end{array}$ \\
\hline
\end{tabular}

and several prefrontal regions. Daianu et al. [100] constructed structural brain networks of 42 patients, $110 \mathrm{MCI}$ patients, and $50 \mathrm{HCs}$ and investigated the rich club organization of networks of the three groups. They found that $\mathrm{AD}$ affected the low-degree brain regions, rather than the rich club comprising the high degree brain regions; global connectivity of $\mathrm{AD}$ was disrupted; the normalized rich club coefficient could be used to detect brain network differences of $\mathrm{MCI} / \mathrm{HC}$ and $\mathrm{AD} / \mathrm{HC}$. To detect abnormal topological organization of structural brain networks of AD patients, Wang et al. [101] used DTI data to construct structural brain networks of $26 \mathrm{AD}$ patients and 16 HCs. They found that although both groups showed small-world property, the AD group exhibited higher small-world index than the HC group. In addition, the AD group displayed decreased global efficiency and local efficiency and increased normalized shortest path length and normalized clustering coefficient.

4.1.2. Functional Brain Networks in AD. Functional brain networks of human beings can be constructed using fMRI and EEG/MEG. In this section, we review recent progress in analyzing the networks based on fMRI and EEG/MEG in AD as shown in Table 3.

(i) Using fMRI: Supekar et al. [88] constructed functional brain networks of $21 \mathrm{AD}$ patients and $18 \mathrm{HCs}$ using task-free fMRI. They found that, in the low frequency band, $0.01-0.05 \mathrm{~Hz}$, the $\mathrm{AD}$ group had lower clustering coefficient than $\mathrm{HCs}$, which cause loss of small-world property in AD. Specifically, they found that clustering coefficients in the left and right hippocampus in AD were lower than $\mathrm{HC}$ while in the left and right precentral gyrus they were not significantly different. To investigate whether aMCI patients disrupt the topological structure of brain networks, Wang et al. [89] constructed functional brain networks of aMCI patients. Compared with $\mathrm{HCs}$, they found decreased functional connectivity and increased path length in the frequency bands, $0.031-0.063 \mathrm{~Hz}$, in aMCI. Brier et al. [85] used path length, clustering coefficient, and modularity to investigate the topology properties of functional brain networks of AD patients (Clinical Dementia Rating $(\mathrm{CDR})=1)$. They constructed different functional brain networks for participants with $\mathrm{CDR}=0$, participants with $\mathrm{CDR}=0.5$, and participants with $\mathrm{CDR}=1$.
They found decreased clustering coefficient and modularity with increasing CDR, but path length was not significantly different. Golbabaei et al. [86] used the local and global measures to assess functional brain networks of AD patients. They found decreased clustering coefficient and global efficiency and increased characteristic path length in AD compared with $\mathrm{HC}$. In addition, $\mathrm{AD}$ patients exhibited decreased node strength, local clustering coefficient, and local efficiency and increased local characteristic path length in olfactory, hippocampus, parahippocampal, amygdala, and superior parietal gyrus.

(ii) Using EEG/MEG: Buldú et al. [34] constructed functional brain networks of MCI patients by using MEG data during a memory task in five frequency bands: $\alpha_{1}(8-11 \mathrm{~Hz}), \alpha_{2}(11-14 \mathrm{~Hz}), \beta_{1}(14-25 \mathrm{~Hz})$, $\beta_{2}(25-35 \mathrm{~Hz})$, and $\gamma(35-45 \mathrm{~Hz})$. They found that the MCI group exhibited an enhancement of the connection strength, which demonstrated that memory processing of MCI patients needs higher energy. In particular, the MCI group also showed lower normalized clustering coefficient and characteristic path length. de Haan et al. [21] used MEG data to explore functional brain network integrity in $\mathrm{AD}$, focusing on network connectivity, synchronizability, and node centrality. They found a loss of network connectivity and altered synchronizability in most frequency bands and demonstrated a low centrality of the left temporal region in the theta band in AD. To clarify these two problems, how functional connectivity is affected in AD subgroups of disease severity and how network hubs change, Engels et al. [103] used EEG data to investigate functional brain networks of three subgroups of $\mathrm{AD}$ patients based on disease severity: mild AD (mi-AD), moderate AD (mo-AD), and severe AD (se-AD). They had three main findings: decreased functional connectivity with increasing $\mathrm{AD}$ severity in the alpha band; increased betweenness centrality with increasing $\mathrm{AD}$ severity in all regions (except for posterior); decreased hub regions in posterior regions and increased hub regions in most anterior regions with increasing $\mathrm{AD}$ severity. To investigate the underlying alteration of the high-level visual (HLV) networks in AD patients, late MCI (LMCI) patients, and early MCI (EMCI) patients, Deng et al. [104] constructed HLV networks of "where" visions across groups. In their study, the 
TABLE 3: Overview of functional brain network studies in AD.

\begin{tabular}{|c|c|c|c|c|c|c|}
\hline Study & Modality & Subjects & $\begin{array}{c}\text { Node } \\
\text { definition }\end{array}$ & Edge definition & $\begin{array}{c}\text { Network } \\
\text { type }\end{array}$ & Main findings \\
\hline $\begin{array}{l}\text { Supekar et al., } \\
2008 \text { [88] }\end{array}$ & fMRI & $\begin{array}{l}21 \mathrm{AD} \\
18 \mathrm{HC}\end{array}$ & $\begin{array}{l}90 \text { regions in } \\
\text { AAL atlas }\end{array}$ & $\begin{array}{c}\text { Wavelet } \\
\text { correlation }\end{array}$ & Weighted & $\begin{array}{l}\text { (1) Compared with } \mathrm{HC} \text {, the loss of } \\
\text { small-world property characterized by a } \\
\text { lower clustering coefficient in } \mathrm{AD} \text { in low } \\
\text { frequency band: } 0.01-0.05 \mathrm{~Hz} \text {. } \\
\text { (2) Lower clustering coefficients in the left } \\
\text { and right hippocampus in } \mathrm{AD} \text {. }\end{array}$ \\
\hline $\begin{array}{l}\text { Wang et al., } \\
2013 \text { [89] }\end{array}$ & fMRI & $\begin{array}{c}37 \mathrm{aMCI} \\
47 \mathrm{HC}\end{array}$ & $\begin{array}{c}1024 \text { regions } \\
\text { in } \mathrm{H}-1024 \\
{[102]}\end{array}$ & $\begin{array}{l}\text { Wavelet } \\
\text { correlation }\end{array}$ & Weighted & $\begin{array}{l}\text { (1) Decreased functional connectivity in the } \\
\text { frequency band, } 0.031-0.063 \mathrm{~Hz} \text {, in aMCI. } \\
\text { (2) Increased path length in aMCI. }\end{array}$ \\
\hline $\begin{array}{l}\text { Brier et al., } 2014 \\
\text { [85] }\end{array}$ & fMRI & $\begin{array}{l}31 \\
\text { CDR } 1 \\
90 \\
\text { CDR } 0.5 \\
205 \\
\text { CDR } 0\end{array}$ & $\begin{array}{l}160 \text { regions } \\
\text { [61] }\end{array}$ & $\begin{array}{l}\text { Pearson } \\
\text { correlation }\end{array}$ & Binary & $\begin{array}{l}\text { (1) Reduced clustering coefficient and } \\
\text { modularity with increasing CDR. } \\
\text { (2) No significant differences in path length } \\
\text { among participants with different CDR. }\end{array}$ \\
\hline
\end{tabular}

(1) Decreased clustering coefficient and global efficiency and increased characteristic path length in AD.

\begin{tabular}{|c|}
\hline $\begin{array}{l}\text { Golbabaei et } \\
\text { al., } 2016[86]\end{array}$ \\
\hline
\end{tabular}

(2) Decreased node strength, local al., 2016 [86 AAL atlas clustering coefficient, and local efficiency and increased local characteristic path length in olfactory, hippocampus, parahippocampal, amygdala, and superior parietal gyrus in $\mathrm{AD}$.

\begin{tabular}{|c|c|c|c|c|c|c|}
\hline $\begin{array}{l}\text { Buldú et al., } \\
2011[34]\end{array}$ & MEG & $\begin{array}{l}19 \mathrm{AD} \\
19 \mathrm{HC}\end{array}$ & 148 sensors & $\begin{array}{c}\text { Synchronization } \\
\text { likelihood }\end{array}$ & Weighted & $\begin{array}{l}\text { (1) Higher connection strength in MCI. } \\
\text { (2) Lower normalized clustering coefficient } \\
\text { and characteristic path length in MCI. }\end{array}$ \\
\hline $\begin{array}{l}\text { de Haan et al., } \\
2012[21]\end{array}$ & MEG & $\begin{array}{l}18 \mathrm{AD} \\
18 \mathrm{HC}\end{array}$ & 149 channels & $\begin{array}{c}\text { Synchronization } \\
\text { likelihood }\end{array}$ & Weighted & $\begin{array}{l}\text { (1) Loss of network connectivity and } \\
\text { altered synchronizability in most frequency } \\
\text { bands in AD. } \\
\text { (2) Low centrality of the left temporal } \\
\text { region in the theta band in AD. }\end{array}$ \\
\hline $\begin{array}{l}\text { Engels et al., } \\
2015 \text { [103] }\end{array}$ & EEG & $\begin{array}{l}117 \\
\text { se-AD } \\
96 \\
\text { mo-AD } \\
105 \\
\text { mi-AD } \\
133 \mathrm{HC}\end{array}$ & 21 channels & Phase lag index & Weighted & $\begin{array}{l}\text { (1) Decreased functional connectivity with } \\
\text { increasing AD severity in the alpha band. } \\
\text { (2) Increased betweenness centrality with } \\
\text { increasing AD severity in all regions } \\
\text { (except for posterior). } \\
\text { (3) Decreased hub regions in posterior } \\
\text { regions and increased hub regions in most } \\
\text { anterior regions with increasing AD } \\
\text { severity. }\end{array}$ \\
\hline $\begin{array}{l}\text { Deng et al., } \\
2016[104]\end{array}$ & EEG & $\begin{array}{l}30 \mathrm{AD} \\
35 \mathrm{LMCI} \\
52 \mathrm{EMCI} \\
44 \mathrm{HC}\end{array}$ & 25 regions & $\begin{array}{c}\text { Wavelet } \\
\text { correlation }\end{array}$ & Binary & $\begin{array}{l}\text { (1) Increased clustering coefficient and } \\
\text { longer characteristic path length in AD } \\
\text { compared with HC. } \\
\text { (2) No significant difference of clustering } \\
\text { coefficient and characteristic path length } \\
\text { between EMCI and HC and between LMCI } \\
\text { and HC. }\end{array}$ \\
\hline
\end{tabular}

AD group showed increased clustering coefficient and longer characteristic path length than the HC group. In addition, compared with the HC group, the LMCI and EMCI groups had no significant difference in terms of clustering coefficient and characteristic path length.
4.2. Schizophrenia. Schizophrenia (https://www.nimh.nih .gov/health/publications/schizophrenia-booklet/index.shtml) (SCZ) is a chronic and severe psychiatric disorder characterized by hallucinations, delusions, and loss of initiative and cognitive dysfunction. Patients with SCZ may seem like losing touch with reality. Families and society are affected by 
SCZ, too. Many patients with SCZ have difficulty in doing a job or caring for themselves, so they rely on others for help. Approximately 8 out of 1,000 individuals have SCZ in their lifetime. In principle, exploring the pathological mechanism of SCZ is a key step in the diagnosis and treatment of SCZ. Some existing studies have demonstrated that the pathological mechanism of SCZ is related to abnormal structural and functional brain networks [47, 160-162].

4.2.1. Structural Brain Networks in SCZ. Structural neuroimaging data, such as sMRI and dMRI, have been widely used in the structural brain network study of SCZ. In this section, we review recent progress in analyzing the structural brain networks based on sMRI and dMRI in SCZ as shown in Table 4.

(i) Using sMRI: Bassett et al. [71] used interregional covariation of gray matter volume as structural connectivity to construct structural brain networks of 259 HCs and 203 SCZ patients. In their study, the cortical cortex was divided into multimodal, unimodal, and transmodal. They found that, in the HC group, the three cortical divisions had small-world property, the multimodal network was hierarchy characterized by frontal hubs with low clustering coefficient, and the transmodal network was assortative. In addition, in the SCZ group, abnormal multimodal network organization showed reduced hierarchy, the loss of frontal and the emergence of nonfrontal hubs, and increased connection distance. Zhang et al. [72] hypothesized that the core symptoms of SCZ originate from the inability to integrate information transmission segregated across different brain regions. To demonstrate this hypothesis, they constructed structural brain networks of SCZ patients using the cortical thickness measurement and found increased characteristic path length and clustering coefficient in the SCZ group compared with the HC group. Moreover, they found reduced nodal centrality in several regions of the default network and increased nodal centrality in primary cortex and paralimbic cortex regions in SCZ. To investigate whether developmental abnormalities associated with SCZ occur in the neonatal stage, Shi et al. [105] constructed morphological brain networks of 26 neonates who were at genetic risk for SCZ and found that although the SCZ group exhibited smallworld topology, the SCZ group had lower global efficiency, longer connection distance, and fewer number of hub nodes with higher betweenness. Tijms et al. [106] constructed more refined structural brain networks of high risk SCZ (HR-SCZ) by using a $6 \times$ $6 \times 6 \mathrm{~mm}^{3}$ cube as a node. They found lower path length in the bilateral inferior frontal gyri, left posterior cingulate region, and superior temporal gyrus and lower clustering coefficient in the right medial superior frontal gyrus, right insula, right fusiform gyrus, left occipital gyrus, and right temporal regions in HR-SCZ compared with HC. (ii) Using dMRI: Zalesky et al. [107] used corticocortical anatomical connectivity at the scale of axonal fiber bundles to construct structural brain networks of 74 SCZ patients and $32 \mathrm{HCs}$. They found that although the SCZ group exhibited small-world topology, the SCZ group had lower global efficiency. In addition, they found lower node degree in medial frontal, parietal/occipital, and the left temporal lobe. To test whether connectivity disturbances are associated with familial vulnerability for SCZ, Collin et al. [108] constructed structural brain networks of 40 SCZ patients, 54 unaffected siblings of SCZ patients, and 51 HCs using DTI data. They found reduced connectivity between rich club hubs across groups, which was lowest in the SCZ group, intermediate in the SCZ siblings group, and highest in the HC group. Moreover, in the SCZ group, they found that lower levels of rich club connectivity were associated with longer duration of illness and worse overall functioning. To investigate alterations in hemispheric white matter (WM) topology in SCZ, Sun et al. [109] constructed weighted hemispheric brain anatomical networks of SCZ patients and HCs. They found that although the hemispheric networks showed smallworld property, the hemispheric-independent deficit of global integration was significantly different in SCZ. Furthermore, compared with the HC group, the SCZ group had longer characteristic path length, lower global efficiency, and reduced asymmetric nodal efficiency in several frontal regions and the hippocampus. Later, Sun et al. [109] continued to investigate alterations in the topological structure of brain anatomical networks using DTI data in SCZ. They constructed weighted brain anatomical networks of $31 \mathrm{SCZ}$ patients and $28 \mathrm{HCs}$ using deterministic tractography and found similar results to the previous study that the SCZ group had smallworld property and that, compared with $\mathrm{HCs}$, the SCZ group had longer characteristic path length and lower global efficiency and was significantly different in the independent deficit of global integration.

4.2.2. Functional Brain Networks in SCZ. fMRI and EEG/ MEG have been widely used in the functional brain network study of SCZ. In this section, we review recent progress in analyzing the functional brain networks based on fMRI and EEG/MEG in SCZ as shown in Table 5.

(i) Using fMRI: Lynall et al. [111] measured aspects of both functional connectivity and functional network topology of SCZ patients to test whether SCZ is a disorder of connectivity. They found decreased strength of functional connectivity and increased diversity of functional connections in SCZ patients. Specifically, they found reduced clustering coefficient and smallworld index, reduced probability of high degree hubs, and increased robustness in the SCZ group. Furthermore, the SCZ group had reduced degree and clustering coefficient in medial parietal, premotor and 
TABLE 4: Overview of structural brain network studies in SCZ.

\begin{tabular}{|c|c|c|c|c|c|}
\hline Study & Modality & Subjects & $\begin{array}{c}\text { Node } \\
\text { definition }\end{array}$ & Edge definition & Network typ \\
\hline $\begin{array}{l}\text { Bassett et al., } \\
2008 \text { [71] }\end{array}$ & sMRI & $\begin{array}{l}203 \mathrm{SCZ} \\
259 \mathrm{HC}\end{array}$ & $\begin{array}{c}104 \text { regions in } \\
\text { Pick atlas }\end{array}$ & $\begin{array}{c}\text { Partial correlation } \\
\text { based on gray } \\
\text { matter volume }\end{array}$ & Binary \\
\hline
\end{tabular}

Main findings

(1) The multimodal network was hierarchy and the transmodal network was assortative in $\mathrm{HC}$.

(2) Abnormal multimodal network organization showed reduced hierarchy, the loss of frontal and the emergence of nonfrontal hubs, and increased connection distance in SCZ.

(1) Increased characteristic path length and clustering coefficient in

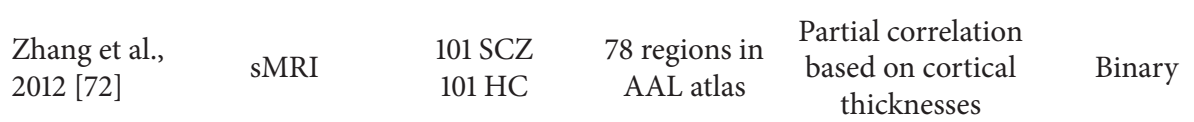

SCZ.

$\begin{array}{lccc}\begin{array}{l}\text { Zhang et al., } \\ 2012[72]\end{array} & \text { sMRI } & 101 \mathrm{SCZ} & 78 \text { regions in } \\ & & \text { AAL atlas } & \begin{array}{c}\text { based on cortic } \\ \text { thicknesses }\end{array}\end{array}$

(2) Reduced nodal centrality in several regions of the default network in SCZ. (3) Increased nodal centrality in primary cortex and paralimbic cortex regions in SCZ.

\begin{tabular}{|c|c|c|c|c|c|}
\hline $\begin{array}{l}\text { Shi et al., } 2012 \\
\text { [105] }\end{array}$ & sMRI & $\begin{array}{l}26 \mathrm{SCZ} \\
26 \mathrm{HC}\end{array}$ & $\begin{array}{l}90 \text { regions in } \\
\text { AAL atlas }\end{array}$ & $\begin{array}{c}\text { Pearson } \\
\text { correlation based } \\
\text { on gray matter } \\
\text { volume }\end{array}$ & Binary \\
\hline
\end{tabular}

(1) Lower global efficiency in SCZ.

(2) Longer connection distance in

SCZ.

(3) Fewer number of hub nodes with higher betweenness in SCZ.

(1) Lower path length in the bilateral inferior frontal gyri, left posterior cingulate region, and superior

$\begin{array}{lccc}\text { Tijms et al., } & 144 & 6 \times 6 \times & \begin{array}{c}\text { The similarity of } \\ \text { grey matter } \\ \text { structure }\end{array}\end{array}$

Binary temporal gyrus in HR-SCZ.
(2) Lower clustering coefficient in the right medial superior frontal gyrus, right insula, right fusiform gyrus, left occipital gyrus, and right temporal regions in HR-SCZ.

\begin{tabular}{|c|c|c|c|c|c|}
\hline $\begin{array}{l}\text { Zalesky et al., } \\
2011 \text { [107] }\end{array}$ & DTI & $\begin{array}{c}74 \mathrm{SCZ} \\
32 \mathrm{HC}\end{array}$ & $\begin{array}{l}82 \text { regions in } \\
\text { AAL atlas }\end{array}$ & FN & Binary \\
\hline
\end{tabular}

(1) Lower global efficiency in SCZ.

(2) Lower node degree in medial frontal, parietal/occipital, and the left temporal lobe in SCZ.

(1) Reduced connectivity between rich club hubs (i.e., lowest in SCZ,

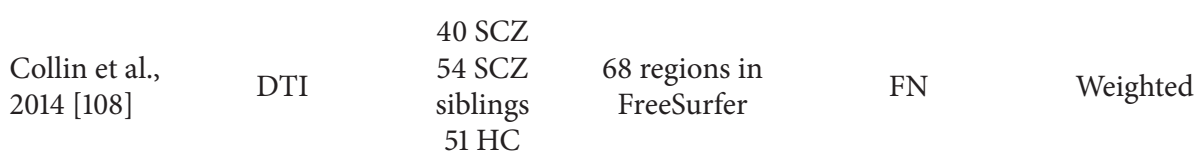
intermediate in SCZ siblings, and highest in $\mathrm{HC}$ ).

(2) Lower levels of rich club connectivity related to longer duration of illness and worse overall functioning.

(1) Significantly different in the hemispheric-independent deficit of global integration in SCZ.

Sun et al., 2015 [109]

DTI

116 SCZ

90 regions in

AAL atlas

FN

(2) Longer characteristic path length and lower global efficiency in SCZ.

(3) Reduced asymmetric nodal efficiency in several frontal regions and the hippocampus in SCZ compared with HC.

(1) Significantly different in the independent deficit of global

Sun et al., 2016 [110]

DTI

31 SCZ

$28 \mathrm{HC}$
$\mathrm{FN} \times \mathrm{FA}$ integration in SCZ compared with HC.

(2) Longer characteristic path length and lower global efficiency in SCZ. 
TABLE 5: Overview of functional brain network studies in SCZ.

\begin{tabular}{|c|c|c|c|c|c|c|}
\hline Study & Modality & Subjects & $\begin{array}{c}\text { Node } \\
\text { definition }\end{array}$ & Edge definition & Network type & Main findings \\
\hline $\begin{array}{l}\text { Lynall et al., } \\
2010 \text { [111] }\end{array}$ & fMRI & $\begin{array}{l}12 \mathrm{SCZ} \\
15 \mathrm{HC}\end{array}$ & $\begin{array}{c}72 \text { regions in } \\
\text { AAL atlas }\end{array}$ & Wavelet correlation & Binary & $\begin{array}{l}\text { (1) Reduced clustering coefficient and } \\
\text { small-world index, reduced probability of } \\
\text { high degree hubs, and increased } \\
\text { robustness in SCZ. } \\
\text { (2) Reduced degree and clustering } \\
\text { coefficient in medial parietal, premotor } \\
\text { and cingulate, and right orbitofrontal } \\
\text { cortical regions in SCZ. }\end{array}$ \\
\hline $\begin{array}{l}\text { Su et al., } 2015 \\
{[112]}\end{array}$ & fMRI & $\begin{array}{l}49 \mathrm{SCZ} \\
28 \mathrm{HC}\end{array}$ & $\begin{array}{l}90 \text { regions in } \\
\text { AAL atlas }\end{array}$ & Pearson correlation & Weighted & $\begin{array}{l}\text { (1) Lower global efficiency in SCZ. } \\
\text { (2) The severity of psychopathology, } \\
\text { negative symptoms, and depression and } \\
\text { anxiety symptoms were related to global } \\
\text { efficiency in SCZ. }\end{array}$ \\
\hline $\begin{array}{l}\text { Hadley et al., } \\
2016 \text { [113] }\end{array}$ & fMRI & $\begin{array}{l}32 \mathrm{SCZ} \\
32 \mathrm{HC}\end{array}$ & $\begin{array}{c}278 \text { regions } \\
{[114]}\end{array}$ & Wavelet correlation & Binary & $\begin{array}{l}\text { (1) Reduced global efficiency and } \\
\text { increased clustering coefficients in SCZ. } \\
\text { (2) Aberrant functional integration and } \\
\text { segregation in SCZ. }\end{array}$ \\
\hline $\begin{array}{l}\text { Ganella et al., } \\
2017 \text { [115] }\end{array}$ & fMRI & $\begin{array}{c}42 \\
\text { TR-SCZ } \\
42 \mathrm{HC}\end{array}$ & $\begin{array}{l}116 \text { regions in } \\
\text { AAL atlas }\end{array}$ & Pearson correlation & Binary & $\begin{array}{l}\text { (1) Reduced global brain functional } \\
\text { connectivity and reduced strength in } \\
\text { frontotemporal, frontooccipital, and } \\
\text { temporooccipital connections in TR-SCZ. } \\
\text { (2) Reduced global efficiency and } \\
\text { increased local efficiency in TR-SCZ. }\end{array}$ \\
\hline $\begin{array}{l}\text { Jhung et al., } \\
2013 \text { [116] }\end{array}$ & EEG & $\begin{array}{l}12 \mathrm{SCZ} \\
13 \mathrm{UHR} \\
13 \mathrm{HC}\end{array}$ & 64 channels & $\begin{array}{l}\text { Synchronization } \\
\text { likelihood }\end{array}$ & Binary & $\begin{array}{l}\text { (1) Reduced small-world property in the } \\
\text { theta band during the working memory } \\
\text { task in SCZ compared with HC. } \\
\text { (2) The small-world index of the UHR } \\
\text { was intermediate value among SCZ, } \\
\text { UHR, and HC during the working } \\
\text { memory task. }\end{array}$ \\
\hline
\end{tabular}
memory task.

\begin{tabular}{|c|c|c|c|c|c|c|}
\hline $\begin{array}{l}\text { Shim et al., } \\
2014 \text { [35] }\end{array}$ & EEG & $\begin{array}{l}34 \mathrm{SCZ} \\
34 \mathrm{HC}\end{array}$ & $\begin{array}{l}314 \text { dipole } \\
\text { sources }\end{array}$ & $\begin{array}{c}\text { Phase locking } \\
\text { value }\end{array}$ & Weighted & $\begin{array}{l}\text { (2) The severity of SCZ symptoms was } \\
\text { negatively correlated with the clustering } \\
\text { coefficient and positively correlated with } \\
\text { path length. }\end{array}$ \\
\hline $\begin{array}{l}\text { Yin et al., } \\
2017 \text { [87] }\end{array}$ & EEG & $\begin{array}{l}14 \\
\text { P-SCZ } \\
14 \\
\text { N-SCZ } \\
14 \mathrm{HC}\end{array}$ & 32 electrodes & $\begin{array}{c}\text { Mutual } \\
\text { information }\end{array}$ & Binary & $\begin{array}{l}\text { (1) Smaller clustering coefficient, larger } \\
\text { average characteristic path length, lower } \\
\text { global efficiency, lower local efficiency, } \\
\text { and smaller degrees in SCZ. } \\
\text { (2) SCZ patients had fewer information } \\
\text { interactions than HCs, and P-SCZ had } \\
\text { more information interactions than } \\
\text { N-SCZ. }\end{array}$ \\
\hline
\end{tabular}

(1) Reduced clustering coefficients and increased path lengths in SCZ.

cingulate, and right orbitofrontal cortical regions. To clarify the correlation between brain network efficiency and SCZ symptoms, Su et al. [112] constructed functional brain networks of 49 SCZ patients and 28 HCs and found that the SCZ group had lower global efficiency than the HC group. Moreover, they also found that the severity of psychopathology, negative symptoms, depression, and anxiety symptoms were related to global efficiency in SCZ. To test whether the balance between functional integration and segregation of brain networks is impaired in SCZ, Hadley et al. [113] constructed functional brain networks of
32 SCZ patients and 32 HCs. They found reduced global efficiency and increased clustering coefficients in SCZ. Since the global efficiency is a measure of functional integration and the clustering coefficient is a measure of functional segregation, their findings demonstrated aberrant functional integration and segregation in SCZ. To explore disruptions in functional connectivity and altered efficiency of functional brain networks in "treatment-resistant" SCZ (TRSCZ), Ganella et al. [115] constructed functional brain networks of 42 TR-SCZ patients and $42 \mathrm{HCs}$. They found reduced global brain functional connectivity 
and reduced strength in frontotemporal, frontooccipital, and temporooccipital connections in TR-SCZ. In addition, they found reduced global efficiency and increased local efficiency in TR-SCZ.

(ii) Using EEG/MEG: Jhung et al. [116] constructed functional brain networks of 13 individuals at ultrahigh risk (UHR) for psychosis, $12 \mathrm{SCZ}$ patients, and $13 \mathrm{HCs}$ to investigate the small-world functional networks across groups. They found that, compared with HCs, SCZ patients had reduced small-world property in the theta band during a working memory task. Furthermore, they found that the small-world index of the UHR during the working memory task showed intermediate value between those of HC and SCZ. Subsequently, Shim et al. [35] investigated smallworld functional networks during auditory oddball tasks and their relations with the severity of symptoms in SCZ. They found reduced clustering coefficients and increased path lengths in SCZ. This finding showed disrupted small-world functional network in SCZ. In addition, the severity of SCZ symptoms was negatively correlated with the clustering coefficient and positively correlated with path length. To test whether positive SCZ (P-SCZ) had more information interaction and negative SCZ (N-SCZ) had less information interaction between brain regions compared with HC, Yin et al. [87] used EEG data to constructed functional brain networks of P-SCZ patients, N-SCZ patients, and HCs. They found smaller clustering coefficient, larger average characteristic path length, lower global efficiency, lower local efficiency, and smaller degrees in SCZ and concluded that SCZ patients had fewer information interactions than HCs, and P-SCZ had more information interactions than N-SCZ.

4.3. Parkinson's Disease. Parkinson's disease (PD) is the second most common progressive neurodegenerative disorder, trailing only $\mathrm{AD}[163,164]$. Advances in neuroimaging techniques and electrophysiological techniques are rapidly expanding the complexity of neurophysiologic understanding of PD. These techniques help to better understand the neurophysiologic mechanisms of PD and its treatments.

4.3.1. Structural Brain Networks in PD. The study of structural brain networks provides another perspective for PD. In this section, we review recent progress in analyzing the structural brain networks based on sMRI and dMRI in PD as shown in Table 6.

(i) Using sMRI: to investigate the topological organization of PD patients, Zhang et al. [117] constructed morphological brain networks of PD patients. In their study, PD patients showed increased global efficiency and local efficiency, increased nodal local efficiency in several regions, decreased local nodal efficiency in several regions, and increased global nodal efficiency in several regions. Pereira et al. [118] used global measures and regional measures to investigate the topology structure of structural brain networks of 123 PD patients and 56 HCs. In their study, to test whether $\mathrm{MCI}$ is associated with disruption in structural brain networks, 123 PD patients were classified into $33 \mathrm{PD}$ patients with $\mathrm{MCI}$ (PD-MCI) and $90 \mathrm{PD}$ patients with $\mathrm{HC}(\mathrm{PD}-\mathrm{HC})$ using the Movement Disorders Society Task Force criteria. They found that, compared with the HC group, the PD-MCI group had reduced connectivity strength between cortical and subcortical regions. In addition, they found that, compared with PD-HC patients and HCs, PD-MCI patients had longer characteristic path length and reduced global efficiency and lower regional efficiency in frontal and parietal regions. Specifically, both PD-MCI and PD-HC had a reorganization of the highly connected regions in the brain networks. To investigate the topological differences of male $\mathrm{PD}$ (PD-M) patients and female PD (PD-F) patients, Yadav et al. [119] constructed structural brain network across groups by using cortical thickness. In their study, compared with PD-F patients, PD-M patients showed lower connectivity strength and clustering coefficients and longer path length. In addition, compared with PD-F patients, PD-M patients exhibited lower nodal betweenness in left caudal middle frontal, left rostral middle frontal, and right parahippocampal regions. Moreover, hubs of the $\mathrm{PD}-\mathrm{M}$ group were right fusiform and right isthmus cingulate region and left inferior temporal and left rostral anterior cingulate, while hubs of the PD-F group were right parahippocampal, right superior temporal, and left rostral middle frontal regions.

(ii) Using dMRI: to reveal topological changes in structural brain networks in PD patients, Li et al. [120] used DTI data to construct structural brain networks of $35 \mathrm{PD}$ patients and $26 \mathrm{HCs}$ by using deterministic tractography. They found that, compared with HCs, PD patients had lower connectivity strength in the feeder and local connections. Furthermore, they found that, in the two modules, the limbic/paralimbic/subcortical module and the cognitive control/attention module, the $\mathrm{PD}$ group had decreased connections compared with the HC group. In addition, they found increased shortest path length and decreased global efficiency in PD. To assess whether structural topological brain network changes are detectable in PD patients, Nigro et al. [121] used centrality, segregation, and integration measures to assess structural brain networks of PD patients. They found decreased network strength, global efficiency, and global clustering coefficient in PD patients compared with HCs. In addition, both groups had 18 hub regions, of which 14 are the same while the other 4 are different.

4.3.2. Functional Brain Networks in PD. The study of functional brain networks provides another perspective for PD. In this section, we review recent progress in analyzing the 
TABLE 6: Overview of structural brain network studies in PD.

\begin{tabular}{|c|c|c|c|c|c|c|}
\hline Study & Modality & Subjects & $\begin{array}{c}\text { Node } \\
\text { definition }\end{array}$ & $\begin{array}{c}\text { Edge } \\
\text { definition }\end{array}$ & Network type & Main findings \\
\hline
\end{tabular}

(1) Increased global efficiency and local efficiency in PD.

(2) Increased nodal local efficiency in the right inferior frontal gyrus (orbital part) and precentral gyrus, left insula and post cingulated cortex, and cerebellum in PD.

\begin{tabular}{|c|c|}
\hline $\begin{array}{l}\text { lang et al., } \\
15 \text { [117] }\end{array}$ & sMRI \\
\hline
\end{tabular}
(3) Decreased local nodal efficiency in the right Heschl's gyrus and precuneus gyrus, and bilateral medial superior frontal gyrus in PD.

(4) Increased global nodal efficiency in the right inferior occipital cortex, inferior frontal gyrus (orbital part), precentral gyrus, and Heschl's gyrus in PD.

(1) Reduced connectivity strength between cortical and subcortical regions in PD-MCI

33

Pereira et al., 2015 [118]

sMRI PD-MCI 90 PD-HC 162 regions in FreeSurfer

Pearson $56 \mathrm{HC}$ compared to HC.

(2) Larger characteristic path length and reduced global efficiency and lower regional efficiency in frontal and parietal regions in the PD-MCI group compared with other two groups.

(3) A reorganization of the highly connected regions in both $\mathrm{PD}-\mathrm{MCI}$ and $\mathrm{PD}-\mathrm{HC}$.

(1) Lower connectivity strength and clustering coefficients and higher path length in PD-M compared with PD-F. (2) Lower nodal betweenness in left caudal middle frontal, left rostral middle frontal, and right parahippocampal regions in PD-M compared with PD-F.

Yadav et al. 43 PD-M $21 \mathrm{PD}-\mathrm{F}$

68 regions in

Pearson $46 \mathrm{HC}$

correlation
Binary
(3) Hubs were right fusiform and right isthmus cingulate region and left inferior temporal and left rostral anterior cingulate in PD-M.

(4) Hubs were right parahippocampal, right superior temporal, and left rostral middle frontal regions in PD-F.

(1) Lower connectivity strength in the feeder and local connections in PD.

(2) Decreased connections in the two modules: the limbic/paralimbic/subcortical module and the cognitive control/attention module in PD.

(3) Increased shortest path length and decreased global efficiency in PD.

(1) Decreased network strength, global efficiency, and global clustering coefficient in PD.

(2) 14 same hub regions and 4 different hub regions between $\mathrm{PD}$ and $\mathrm{HC}$. functional brain networks based on fMRI and EEG/MEG in $\mathrm{PD}$ as shown in Table 7.

(i) Using fMRI: to investigate the efficiency of functional brain networks of PD patients, Skidmore et al. [122] constructed functional brain networks of 14
PD patients and 15 HCs. They found decreased global efficiency and decreased nodal efficiency in the left supplementary motor cortex, contiguous precentral regions, the calcarine cortices, secondary visual regions, and the certain regions within the cerebellum in PD patients. Later, to investigate altered 
TABLE 7: Overview of functional brain network studies in PD.

\begin{tabular}{lccccc}
\hline Study & Modality & Subjects & $\begin{array}{c}\text { Node } \\
\text { definition }\end{array}$ & $\begin{array}{c}\text { Edge } \\
\text { definition }\end{array}$ & Network type \\
\hline Skidmore et & fMRI & $14 \mathrm{PD}$ & 116 regions in & Wavelet & Weighted \\
al., 2011 [122] & 15 HC & AAL atlas & correlation & W
\end{tabular}

(1) Decreased global efficiency in PD.

(2) Decreased nodal efficiency the left supplementary motor cortex, contiguous precentral regions, the calcarine cortices, secondary visual regions, and the certain regions within the cerebellum in PD.

(1) Lower global efficiency in PD in PD.

(2) Increased connectivity within the sensorimotor network and decreased interaction of the visual network with other brain modules in PD.

\begin{tabular}{|c|c|c|c|c|c|c|}
\hline $\begin{array}{l}\text { Göttlich et } \\
\text { al., } 2013 \text { [123] }\end{array}$ & fMRI & $\begin{array}{l}37 \mathrm{PD} \\
20 \mathrm{HC}\end{array}$ & 343 regions & $\begin{array}{l}\text { Zero-lag } \\
\text { Pearson } \\
\text { correlation }\end{array}$ & Binary & $\begin{array}{l}\text { (3) Lower connectivity between the } \\
\text { cuneus and the ventral caudate, medial } \\
\text { orbitofrontal cortex, and the temporal }\end{array}$ \\
\hline
\end{tabular}

(3) Lower connectivity between the

(4) Decreased degree in the occipital lobe and increased degree in the superior parietal cortex, posterior cingulate gyrus, supramarginal gyrus, and supplementary motor area.

(1) Lower clustering coefficient and local efficiency in PD.

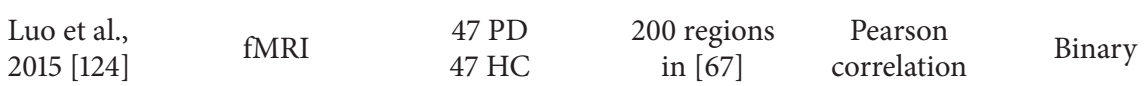

(2) Reduced node centralities and connectivity strength in temporal-occipital and sensorimotor regions in $\mathrm{PD}$.

(1) Higher nodal degree in the right and left dorsolateral prefrontal cortex in PD.

$\begin{array}{lccccc}\text { Koshimori et } & \text { fMRI } & 42 \mathrm{PD} & 120 \text { regions in } & \begin{array}{c}\text { Pearson } \\ \text { correlation }\end{array} & \text { Binary } \\ \text { al., 2016 [125] } & & 23 \mathrm{HC} & {[61]} & \text {. }\end{array}$

(2) Reduced local efficiency the right mid-insula in PD.

(3) Reduced nodal betweenness centrality in the right presupplementary motor area in PD.

(1) Lower local clustering coefficient with preserved path length in the delta

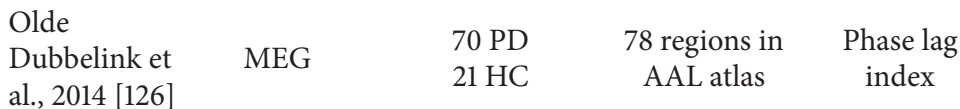
frequency band in PD.

al., 2014 [126]

$21 \mathrm{HC}$

AAL atlas

index

Weighted

(2) Decreased local clustering coefficient in multiple frequency bands with decreased path length in the alpha2 frequency band in PD.

(1) Higher connectivity strength in the theta band in PD-HC compared with HC. (2) higher gamma, lambda, and modularity in PD-HC compared with HC.

$\begin{array}{lcccc}\text { Utianski et } & \text { PD-D } & & \text { Phase lag } & \text { Weighted } \\ \text { al., 2016 [127] } & \text { EEG } & 57 & \text { index } & \\ & & \text { PD-HC } & & \\ & 57 \mathrm{HC} & \end{array}$

(3) lower functional connectivity in the alphal band in PD-D compared with PD-HC.

(4) Lower gamma and lambda in the alphal band and higher modularity in both alpha bands in PD-D compared with PD-HC.

brain functional connectivity of PD patients, Göttlich et al. [123] constructed functional brain networks of $37 \mathrm{PD}$ patients and $20 \mathrm{HCs}$. They found lower global efficiency in PD. Moreover, by analyzing brain network modules, they found out only increased connectivity within the sensorimotor network and decreased interaction of the visual network with other brain modules but also lower connectivity 
between the cuneus and the ventral caudate, medial orbitofrontal cortex, and the temporal lobe in PD. In addition, they found decreased degree in the occipital lobe and increased degree in the superior parietal cortex, posterior cingulate gyrus, supramarginal gyrus, and supplementary motor area in PD. Subsequently, Luo et al. [124] continued to investigate the topological organization of functional brain networks of PD patients. They found lower clustering coefficient and local efficiency in PD patients compared with HCs. Moreover, they found reduced node centrality and connectivity strength in temporal-occipital and sensorimotor regions in PD patients. To investigate functional changes in cognitive and sensorimotor networks in PD patients, Koshimori et al. [125] used nodal degree, local efficiency, and betweenness centrality measures to assess functional brain networks of PD patients. They found that, compared with HCs, PD patients showed higher nodal degree in the right and left dorsolateral prefrontal cortex, reduced local efficiency the right mid-insula, and reduced nodal betweenness centrality in the right presupplementary motor area.

(ii) Using EEG/MEG: to explore the spatial organization of alterations in functional connectivity between brain regions, Olde Dubbelink et al. [126] used clustering coefficient and shortest path length to measure functional brain networks of $70 \mathrm{PD}$ patients and 21 HCs. They found lower local clustering coefficient with preserved path length in the delta frequency band. Moreover, they found decreased local clustering coefficient in multiple frequency bands with decreased path length in the alpha2 frequency band. To determine the differences between PD patients who are healthy control (PD-HC) and HCs and between PD-HC and PD dementia (PD-D), Utianski et al. [127] used EEG data to construct functional brain networks across groups. In their study, compared with HCs, PD-HC patients showed higher connectivity strength in the theta band but no differences in other frequency bands. Moreover, PD-HC patients exhibited higher gamma, lambda, and modularity than HCs. Compared with the PD-HC group, the PD-D group showed lower functional connectivity, gamma, and lambda in the alphal band and higher modularity in both alpha bands.

4.4. Multiple Sclerosis. Multiple sclerosis (MS) is an inflammatory and degenerative disease of the central nervous system (CNS). It is characterized by multiple lesions mainly affecting the WM, accompanying structural and functional disconnection between various regions in the CNS, resulting in kinds of signs and symptoms.

4.4.1. Structural Brain Networks in MS. The study of structural brain networks provides another perspective for MS. In this section, we review recent progress in analyzing the structural brain networks based on sMRI and dMRI in MS as shown in Table 8 .

(i) Using sMRI: to investigate the correlation between the WM lesion load and the topological efficiency of structural brain networks in MS patients, He et al. [128] divided all MS patients into six subgroups based on corresponding total WM lesion loads (TWMLL) and constructed structural brain networks across groups. They found decreased integrated absolute local and global efficiency and decreased integrated relative local efficiency with increasing TWMLL in MS. Tewarie et al. [129] constructed structural brain networks based on cortical thicknesses to investigate the topological differences of MS patients and HCs. They found higher normalized clustering coefficient and higher normalized shortest path length in MS.

(ii) Using dMRI: to investigate the alterations in the topological organization of the WM structural networks, Shu et al. [130] used DTI and deterministic tractography to constructed the WM structural networks of 39 MS patients and 39 HCs. They found that the MS patients and the HCs showed efficient small-world property in their WM structural networks. Moreover, compared with HCs, MS patients had decreased global efficiency and decreased local efficiency in the sensorimotor, visual, default-mode, and language regions. Later, Shu et al. [132] continued to investigate the topological alterations of structural networks in 41 clinically isolated syndrome (CIS) patients, $32 \mathrm{MS}$ patients, and $35 \mathrm{HCs}$. They found that, compared with HCs, both CIS and MS patients showed decreased network strength, global and local efficiency, and clustering coefficient and increased shortest path length. Moreover, compared with the HCs, the MS patients exhibited increased gamma and sigma, and, compared with the CIS patients, the MS patients exhibited reduced network strength and global and local efficiency and increased shortest path length, gamma, and sigma. To explore the underlying brain mechanisms of major depression MS (MD-MS) patients and nondepressed MS (ND-MS) patients, Nigro et al. [131] used DTI data to construct structural brain networks across groups. In their study, both MS patient groups showed small-world property. In addition, MS patients exhibited increased path length compared with HCs, and MD-MS patients showed increased local path length in the right hippocampus and right amygdala compared with ND-MS patients and HCs. To investigate changes in structural connectivity in MS, Llufriu et al. [133] used FA values as connectivity strength between brain regions to construct structural brain networks of $72 \mathrm{MS}$ patients and 38 HCs. In their study, compared with HCs, MS patients showed decreased transitivity and global efficiency and increased path length. Moreover, MS patients displayed decreased nodal strength in 26 of 84 gray matter regions and increased betweenness centrality in right pallidum and left insula. 
TABLE 8: Overview of structural brain network studies in MS.

\begin{tabular}{|c|c|c|c|c|c|c|}
\hline Study & Modality & Subjects & $\begin{array}{c}\text { Node } \\
\text { definition }\end{array}$ & Edge definition & Network type & Main findings \\
\hline $\begin{array}{l}\text { He et al., } \\
2009 \text { [128] }\end{array}$ & sMRI & $\begin{array}{c}102 \mathrm{MS} \\
42 \mathrm{HC}\end{array}$ & 54 regions & $\begin{array}{l}\text { Pearson } \\
\text { correlation } \\
\text { based on } \\
\text { cortical } \\
\text { thicknesses }\end{array}$ & Binary & $\begin{array}{l}\text { (1) Decreased integrated absolute local } \\
\text { and global efficiency with increasing } \\
\text { TWMLL in MS. } \\
\text { (2) Decreased integrated relative local } \\
\text { efficiency with increasing TWMLL in MS. }\end{array}$ \\
\hline $\begin{array}{l}\text { Tewarie et al., } \\
2014 \text { [129] }\end{array}$ & sMRI & $\begin{array}{c}102 \mathrm{MS} \\
42 \mathrm{HC}\end{array}$ & $\begin{array}{c}78 \text { regions in } \\
\text { AAL atlas }\end{array}$ & $\begin{array}{l}\text { Pearson } \\
\text { correlation } \\
\text { based on } \\
\text { cortical } \\
\text { thicknesses }\end{array}$ & Weighted & $\begin{array}{l}\text { (1) Higher normalized clustering } \\
\text { coefficient in MS. } \\
\text { (2) Higher normalized shortest path } \\
\text { length in MS. }\end{array}$ \\
\hline $\begin{array}{l}\text { Shu et al., } \\
2011 \text { [130] }\end{array}$ & DTI & $\begin{array}{l}39 \mathrm{MS} \\
39 \mathrm{HC}\end{array}$ & $\begin{array}{c}90 \text { regions in } \\
\text { AAL atlas }\end{array}$ & FN & Weighted & $\begin{array}{l}\text { (1) Decreased global efficiency in MS. } \\
\text { (2) Decreased local efficiency in the } \\
\text { sensorimotor, visual, default-mode, and } \\
\text { language areas in MS. }\end{array}$ \\
\hline $\begin{array}{l}\text { Nigro et al., } \\
2015 \text { [131] }\end{array}$ & DTI & $\begin{array}{c}20 \\
\text { MD-MS } \\
22 \\
\text { ND-MS } \\
16 \text { HC }\end{array}$ & $\begin{array}{l}90 \text { regions in } \\
\text { AAL atlas }\end{array}$ & FN & Weighted & $\begin{array}{l}\text { (1) Increased path length in MS patients } \\
\text { compared with HCs. } \\
\text { (2) Increased local path length in the } \\
\text { right hippocampus and right amygdala in } \\
\text { MD-MS compared with ND-MS and HC. }\end{array}$ \\
\hline $\begin{array}{l}\text { Shu et al., } \\
2016 \text { [132] }\end{array}$ & DTI & $\begin{array}{l}41 \mathrm{CIS} \\
32 \mathrm{MS} \\
35 \mathrm{HC}\end{array}$ & $\begin{array}{l}90 \text { regions in } \\
\text { AAL atlas }\end{array}$ & FN & Weighted & $\begin{array}{l}\text { (1) Decreased network strength, global } \\
\text { and local efficiency, and clustering } \\
\text { coefficient and increased shortest path } \\
\text { length in both CIS and MS. } \\
\text { (2) Increased gamma and sigma in MS } \\
\text { compared with HC. } \\
\text { (3) Reduced network strength and global } \\
\text { and local efficiency and increased } \\
\text { shortest path length, gamma, and sigma } \\
\text { in MS compared with CIS. }\end{array}$ \\
\hline $\begin{array}{l}\text { Llufriu et al., } \\
2017 \text { [133] }\end{array}$ & DTI & $\begin{array}{l}72 \mathrm{MS} \\
38 \mathrm{HC}\end{array}$ & $\begin{array}{c}84 \text { regions in } \\
\text { FreeSurfer }\end{array}$ & FA & Weighted & $\begin{array}{l}\text { (1) Decreased transitivity and global } \\
\text { efficiency and increased path length in } \\
\text { MS. } \\
\text { (2) Increased betweenness centrality in } \\
\text { right pallidum and left insula in MS. } \\
\text { (3) Decreased nodal strength in } 26 \text { of the } \\
84 \text { brain regions in MS. }\end{array}$ \\
\hline
\end{tabular}

4.4.2. Functional Brain Networks in MS. The study of functional brain networks provides another perspective for MS. In this section, we review recent progress in analyzing the functional brain networks based on fMRI and EEG/MEG in MS as shown in Table 9.

(i) Using fMRI: to investigate the modularity of MS patients, Gamboa et al. [134] constructed functional brain networks of MS patients and found increased modularity in MS patients compared with HCs. To explore the topological organization of functional brain network connectivity, Rocca et al. [135] constructed functional brain networks of 246 MS patients and 55 HCs. They found that, compared with HCs, MS patients lost hubs in the superior frontal gyrus and precuneus and anterior cingulum in the left hemisphere and showed new hubs in the left temporal pole and cerebellum, located at different hemisphere for basal ganglia hubs. Furthermore, MS patients exhibited decreased nodal degree in the bilateral caudate nucleus and right cerebellum. Shu et al. [132] constructed functional networks in CIS patients, MS patients, and HCs to investigate the topological alterations across groups. They found that, compared with the HCs, the MS patients showed decreased local efficiency and clustering coefficient. Moreover, they found that the CIS group had no significant differences with the other two groups in any global metrics. Later, Liu et al. [136] continued to investigate the topological organization of CIS patients and MS patients. They found that CIS patients showed intermediate global efficiency between MS patients and HCs, and global efficiency of MS patients was the lowest. In addition, MS patients exhibited lower local efficiency than HCs.

(ii) Using EEG/MEG: to investigate functional connectivity changes in MS, Schoonheim et al. [137] used MEG data to construct functional brain networks of MS patients. In their study, compared with HCs, MS 
TABLE 9: Overview of functional brain network studies in MS.

\begin{tabular}{lcccccc}
\hline Study & Modality & Subjects & $\begin{array}{c}\text { Node } \\
\text { definition }\end{array}$ & Edge definition & Network type & Main findings \\
\hline $\begin{array}{l}\text { Gamboa et } \\
\text { al., 2014 [134] }\end{array}$ & fMRI & $\begin{array}{l}16 \mathrm{MS} \\
20 \mathrm{HC}\end{array}$ & $\begin{array}{c}116 \text { regions in } \\
\text { AAL atlas }\end{array}$ & $\begin{array}{c}\text { Pearson } \\
\text { correlation }\end{array}$ & Binary & (1) Increased modularity in MS. \\
\hline
\end{tabular}

(1) Lost hubs in superior frontal gyrus,

(1) Lost hubs in superior frontal gyrus,
precuneus, and anterior cingulum in the left hemisphere in MS.

\begin{tabular}{|c|c|c|c|c|}
\hline $\begin{array}{l}\text { Rocca et al., } \\
2016 \text { [135] }\end{array}$ & fMRI & $\begin{array}{c}246 \mathrm{MS} \\
55 \mathrm{HC}\end{array}$ & $\begin{array}{l}116 \text { regions in } \\
\text { AAL atlas }\end{array}$ & $\begin{array}{l}\text { Pearson } \\
\text { correlation }\end{array}$ \\
\hline
\end{tabular}

(2) New hubs in the left temporal pole and cerebellum in MS.
(3) Different hemisphere for basal ganglia hubs in MS. (4) Decreased nodal degree in the
bilateral caudate nucleus and right
cerebellum in MS.

\begin{tabular}{|c|c|c|c|c|c|c|}
\hline $\begin{array}{l}\text { Shu et al., } \\
2016 \text { [132] }\end{array}$ & fMRI & $\begin{array}{l}41 \mathrm{CIS} \\
32 \mathrm{MS} \\
35 \mathrm{HC}\end{array}$ & $\begin{array}{l}90 \text { regions in } \\
\text { AAL atlas }\end{array}$ & $\begin{array}{l}\text { Pearson } \\
\text { correlation }\end{array}$ & Weighted & $\begin{array}{l}\text { (1) Decreased local efficiency and } \\
\text { clustering coefficient in MS compared } \\
\text { with HC. } \\
\text { (2) No significant differences with the } \\
\text { other two groups in any global metrics in } \\
\text { the CIS group. }\end{array}$ \\
\hline $\begin{array}{l}\text { Liu et al., } \\
2017 \text { [136] }\end{array}$ & fMRI & $\begin{array}{l}35 \mathrm{CIS} \\
37 \mathrm{MS} \\
36 \mathrm{HC}\end{array}$ & $\begin{array}{l}90 \text { regions in } \\
\text { AAL atlas }\end{array}$ & $\begin{array}{l}\text { Pearson } \\
\text { correlation }\end{array}$ & Weighted & $\begin{array}{l}\text { (1) CIS showed intermediate global } \\
\text { efficiency between MS and HC. } \\
\text { (2) Lower global efficiency and local } \\
\text { efficiency in MS compared with HC. }\end{array}$ \\
\hline $\begin{array}{l}\text { Schoonheim } \\
\text { et al., } 2013 \\
{[137]}\end{array}$ & MEG & $\begin{array}{l}34 \mathrm{MS} \\
28 \mathrm{HC}\end{array}$ & 137 channels & $\begin{array}{l}\text { Synchronization } \\
\text { likelihood }\end{array}$ & Weighted & $\begin{array}{l}\text { (1) Increased connectivity strength in } \\
\text { theta, lower alpha, and beta bands in MS. } \\
\text { (2) Decreased connectivity strength in } \\
\text { the upper alpha band in MS. } \\
\text { (3) Increased path length and clustering } \\
\text { coefficient in the lower alpha band in MS. }\end{array}$ \\
\hline $\begin{array}{l}\text { Tewarie et al., } \\
2014 \text { [129] }\end{array}$ & MEG & $\begin{array}{l}102 \mathrm{MS} \\
42 \mathrm{HC}\end{array}$ & $\begin{array}{l}78 \text { regions in } \\
\text { AAL atlas }\end{array}$ & Phase lag index & Weighted & $\begin{array}{l}\text { (1) Higher normalized path length in the } \\
\text { theta band in MS. } \\
\text { (2) Lower normalized clustering } \\
\text { coefficient in the alpha } 2 \text { band in MS. }\end{array}$ \\
\hline
\end{tabular}

patients showed increased functional connectivity strength in theta, lower alpha, and beta bands and decreased functional connectivity strength in the upper alpha band. Furthermore, MS patients exhibited increased path length and clustering coefficient in the lower alpha band compared with HCs. Later, Tewarie et al. [129] constructed functional brain networks of MS patients and HCs to investigate the topological differences across groups. They found higher normalized path length in the theta band and lower normalized clustering coefficient in the alpha2 band in MS.

In addition to the above four brain disorders, brain network analysis has also been applied to other brain disorders, such as attention deficit/hyperactivity (ADHD) [165-167], epilepsy [168-170], and autism [171-173].

\section{Conclusions and Outlook}

In summary, the development of noninvasive neuroimaging and electrophysiological techniques (such as sMRI, dMRI, fMRI, and EEG/MEG) has enabled us to construct human brain structural and functional connectivity networks, while the complex network analysis has revealed a number of important topological properties hidden in the human brain structural and functional networks, such as small-world property, modularity, hubs, and rich club. The study of complex brain networks will not only promote the construction of the human brain connectome but also deepen our understanding of the important issues such as the information processing mode of the brain and the working mechanism of various cognitive functions. Moreover, to explore the brain network topology caused by brain disorder abnormal changes, the methods with brain network analysis have been applied to different brain disorder studies, such as the above-mentioned four brain disorders in Section 4. Brain network analysis not only provides a new perspective for revealing the pathophysiologic mechanisms of brain disorders at the system level, but also establishes some brain network neuroimaging and electrophysiological markers to describe different brain disorders. For example, for the abovementioned four brain disorders in Section 4, most findings indicate that, compared with the HC group, the disorder group exhibited decreased small-world index and decreased global efficiency at the global level, while, at the local level, the 
disorder group showed the loss of hub nodes and decreased local efficiency compared with the HC group. Therefore, brain network analysis can provide important auxiliary guidance for early diagnosis and treatment of brain disorders.

As can be seen from Section 4, although many findings are obtained for a specific brain disorder after using brain network analysis, parts of findings obtained by different studies are not consistent or even opposite. For example, Tijms et al. [96] found that, compared with the HC group, the AD group exhibited decreased small-world index, while Wang et al. [101] found that the AD group showed higher small-world index compared with the HC group; Lynall et al. [111] found that, compared with the HC group, the SCZ group exhibited decreased clustering coefficient, while Hadley et al. [113] found that the SCZ group showed increased clustering coefficient compared with the HC group. One of the main causes of the above similar opposite results is that the experimental data is too small. However, although the results of the different studies are opposite, a single property cannot be used to determine whether there is a difference between the disorder group and the HC group in the field of brain network analysis. In general, in order to determine the difference between the disorder group and the HC group, multiple properties must be used to complete it. Hence, in order to obtain more accurate brain network analysis results, there are many problems and challenges to be solved urgently as follows:

(i) How to construct a brain network that conforms to the working mechanism of the brain is a primary problem in brain network analysis. In Section 2, we introduce two basic elements of the brain network, nodes and edges, and present a variety of their common definitions, such as node definitions based on different brain atlas and edge definitions based on different correlations between two nodes. The various existing node definitions in constructing brain networks can only reflect one aspect of the brain regions themselves, such as cortical thickness and time series. Similarly, a specific edge definition can also only reflect one aspect of the connectivity between the brain regions, such as the number of fibers and Pearson correlation. Thus, how to evaluate the impact of different nodes and edges on the brain network and determine the most reasonable definition of nodes and edges is a key problem, as well as an important challenge in constructing the brain network.

(ii) Studies have shown that the brain structural and functional network topology of most brain disorders have abnormal changes, but there is still no unified conclusion on the trends and amplitudes of the brain network topology properties of various brain disorders. For example, Shu et al. [132] used DTI and fMRI to investigate the brain network topology properties of MS, and the results are inconsistent as shown in Table 5. Thus, it is urgent to solve the problem of how to integrate multimodal data to analyze and understand the pathophysiologic mechanisms of brain disorders, and establish reliable and effective neuroimaging and electrophysiological diagnostic markers in brain network research.

(iii) The structure and function of a brain are inseparable; the structure is the basis of function and the function is the representation of the structure. It has been shown that the structure and function of the human brains are closely related [174, 175]. Thus, it is challenging to combine the structure network and function network of a brain for evaluating the similarity and specificity of brain structure-function network comprehensively and understanding the effect of structural network organization on brain function formation and brain function shaping on brain structure.

(iv) The functional activity of a brain is a dynamic process, and most existing functional networks only describe the topological properties of brain function activity in a certain period of time. Thus, how to construct a dynamic brain function network to find the regularity of the brain function topology properties with time changes in a smaller time scale is one of the directions of future brain network research. It can further explore the mechanisms of brain real-time functional activities.

(v) Because of their simplicity, many researchers focused on the undirected brain networks. However, the information transmission of each activity of a brain is directional, and the undirected network analysis is unable to obtain the results with the direction of information flow. As the undirected network analysis cannot reveal the direction of information transmission hidden in the brain structural and functional networks, it cannot really reflect the real brain activities. In order to reflect the real brain activities and for more in-depth understanding of the regularity of the brain structural organization patterns and functional activities, it is necessary to construct the directional structural and functional brain networks to understand the brain activities and to further reveal how to transmit information in the brain activities. How to construct effective directed brain networks and how to carry out effective directed brain network analysis are two important problems and challenges for researchers.

(vi) As can be seen from several tables (Tables 2-9), the sample sizes of most existing brain network researches are too small, most of which are not more than 100. Therefore, the results of brain network analysis may be incomplete, or even wrong, which to some extent restricts the development of brain network analysis. Thus, in order to make the results of brain network analysis closer to reality, the sample size of the experimental data should be urgently expanded.

(vii) Most brain network studies are based on a single layer network. More recently, the multilayer network studies [176-178] have also been proposed. Since 
multilayer networks can integrate different anatomical/functional types of links or different frequency bands, the multilayer network studies are likely to become one of the most promising future research directions.

Study of brain networks is a part of brain science, which incorporates a wide range of disciplines such as neuroscience and graph theory. It is reasonable to expect that brain network analysis would bring more remarkable achievements in the near future.

\section{Conflicts of Interest}

The authors declare that there are no conflicts of interest regarding the publication of this paper.

\section{Acknowledgments}

The authors would like to express their gratitude for the support from the National Natural Science Foundation of China under Grant nos. 61232001, 61420106009, and 61622213.

\section{References}

[1] F. A. C. Azevedo, L. R. B. Carvalho, L. T. Grinberg et al., "Equal numbers of neuronal and nonneuronal cells make the human brain an isometrically scaled-up primate brain," Journal of Comparative Neurology, vol. 513, no. 5, pp. 532-541, 2009.

[2] S. Herculano-Houzel, "The human brain in numbers: a linearly scaled-up primate brain," Frontiers in Human Neuroscience, vol. 3, article 31, 2009.

[3] M. P. van den Heuvel, R. S. Kahn, J. Goñi, and O. Sporns, "Highcost, high-capacity backbone for global brain communication," Proceedings of the National Acadamy of Sciences of the United States of America, vol. 109, no. 28, pp. 11372-11377, 2012.

[4] O. Sporns, "The human connectome: Origins and challenges," NeuroImage, vol. 80, pp. 53-61, 2013.

[5] M. G. Mattar and D. S. Bassett, Brain network architecture: Implications for human learning, preprint arXiv:1609.01790.

[6] J. Lehrer, "Neuroscience: Making connections," Nature, vol. 457, no. 7229, pp. 524-527, 2009.

[7] O. Sporns, D. R. Chialvo, M. Kaiser, and C. C. Hilgetag, "Organization, development and function of complex brain networks," Trends in Cognitive Sciences, vol. 8, no. 9, pp. 418-425, 2004.

[8] J. D. Power, D. A. Fair, B. L. Schlaggar, and S. E. Petersen, “ The development of Human Functional Brain Networks," Neuron, vol. 67, no. 5, pp. 735-748, 2010.

[9] O. Sporns, "The human connectome: a complex network," Annals of the New York Academy of Sciences, vol. 1224, no. 1, pp. 109-125, 2011.

[10] T. E. J. Behrens and O. Sporns, "Human connectomics," Current Opinion in Neurobiology, vol. 22, no. 1, pp. 144-153, 2012.

[11] D. C. van Essen, S. M. Smith, D. M. Barch, T. E. J. Behrens, E. Yacoub, and K. Ugurbil, "The WU-Minn human connectome project: an overview," NeuroImage, vol. 80, pp. 62-79, 2013.

[12] D. S. Bassett and O. Sporns, "Network neuroscience," Nature Neuroscience, vol. 20, no. 3, pp. 353-364, 2017.

[13] M.-M. Poo, J.-L. Du, N. Y. Ip, Z.-Q. Xiong, B. Xu, and T. Tan, "China brain project: basic neuroscience, brain diseases, and brain-inspired computing," Neuron, vol. 92, no. 3, pp. 591-596, 2016.

[14] O. Sporns, "From simple graphs to the connectome: networks in neuroimaging," Neurolmage, vol. 62, no. 2, pp. 881-886, 2012.

[15] Y. D. Reijmer, A. Leemans, K. Caeyenberghs, S. M. Heringa, H. L. Koek, and G. J. Biessels, "Disruption of cerebral networks and cognitive impairment in Alzheimer disease," Neurology, vol. 80, no. 15, pp. 1370-1377, 2013.

[16] J. Liu, M. Li, J. Wang, F. Wu, T. Liu, and Y. Pan, "A survey of MRIbased brain tumor segmentation methods," Tsinghua Science and Technology, vol. 19, no. 6, pp. 578-595, 2014.

[17] M. E. Shenton, M. Kubicki, and N. Makris, "Understanding alterations in brain connectivity in attention-deficit/hyperactivity disorder using imaging connectomics," Biological Psychiatry, vol. 76, no. 8, pp. 601-602, 2014.

[18] J. Liu, M. Li, W. Lan, F. Wu, Y. Pan, and J. Wang, "Classification of Alzheimer's disease using whole brain hierarchical network," IEEE Transactions on Computational Biology and Bioinformatics, no. 99, 2016.

[19] C. Garcia-Ramos, J. J. Lin, T. S. Kellermann, L. Bonilha, V. Prabhakaran, and B. P. Hermann, "Graph theory and cognition: A complementary avenue for examining neuropsychological status in epilepsy," Epilepsy \& Behavior, vol. 64, pp. 329-335, 2016.

[20] J. Liu, J. Wang, Z. Tang, B. Hu, F. Wu, and Y. Pan, "Improving Alzheimeres disease classification by combining multiple measures," IEEE Transactions on Computational Biology and Bioinformatics, 2017.

[21] W. de Haan, W. M. van der, H. Flier, P. F. Van Mieghem, P. Scheltens, and C. J. Stam, "Disruption of functional brain networks in alzheimers disease: what can we learn from graph spectral analysis of resting-state magnetoencephalography?" Brain connectivity, vol. 2, no. 2, pp. 45-55, 2012.

[22] J. Liu, M. Li, Y. Pan, F. Wu, X. Chen, and J. Wang, "Classification of Schizophrenia based on individual hierarchical brain networks constructed from structural MRI images," IEEE Transactions on NanoBioscience, 2017.

[23] A. Fornito, J. Yoon, A. Zalesky, E. T. Bullmore, and C. S. Carter, "General and specific functional connectivity disturbances in first-episode schizophrenia during cognitive control performance," Biological Psychiatry, vol. 70, no. 1, pp. 64-72, 2011.

[24] J. Liu, J. Wang, B. Hu, F. Wu, and Y. Pan, "Alzheimer's disease classification based on individual hierarchical networks constructed with 3-D," IEEE Transactions on NanoBioscience, vol. 16, no. 6, pp. 1-10, 2017.

[25] P. Skudlarski, K. Jagannathan, K. Anderson et al., "Brain connectivity is not only lower but different in schizophrenia: a combined anatomical and functional approach," Biological Psychiatry, vol. 68, no. 1, pp. 61-69, 2010.

[26] J. Liu, Y. Pan, M. Li et al., "Applications of deep learning to mri images: a survey," Big Data Mining and Analytics, vol. 1, no. 1, 2017.

[27] E. M. Haacke, R. W. Brown, M. R. Thompson et al., Magnetic Resonance Imaging: Physical Principles and Sequence Design, vol. 82, Wiley-Liss, New York, NY, USA, 1999.

[28] S. Mori, B. J. Crain, V. P. Chacko, and P. C. M. Van Zijl, “Threedimensional tracking of axonal projections in the brain by magnetic resonance imaging," Annals of Neurology, vol. 45, no. 2, pp. 265-269, 1999.

[29] S. A. Huettel, A. W. Song, and G. McCarthy, Functional Magnetic Resonance Imaging, vol. 1, Sinauer Associates, Sunderland, Mass, USA, 2004. 
[30] N. K. Logothetis, "What we can do and what we cannot do with fMRI," Nature, vol. 453, no. 7197, pp. 869-878, 2008.

[31] E. Niedermeyer and F. L. da Silva, Electroencephalography: Basic Principles, Clinical Applications, and Related Fields, Lippincott Williams \& Wilkins, 2005.

[32] M. Hämäläinen, R. Hari, R. J. Ilmoniemi, J. Knuutila, and O. V. Lounasmaa, "Magnetoencephalography-theory, instrumentation, and applications to noninvasive studies of the working human brain," Reviews of Modern Physics, vol. 65, no. 2, pp. 413497, 1993.

[33] W. de Haan, Y. A. L. Pijnenburg, R. L. M. Strijers et al., "Functional neural network analysis in frontotemporal dementia and Alzheimer's disease using EEG and graph theory," BMC Neuroscience, vol. 10, no. 101, 2009.

[34] J. M. Buldú, R. Bajo, F. Maestú et al., "Reorganization of functional networks in mild cognitive impairment," PLOS ONE, vol. 6, no. 5, Article ID e19584, 2011.

[35] M. Shim, D.-W. Kim, S.-H. Lee, and C.-H. Im, "Disruptions in small-world cortical functional connectivity network during an auditory oddball paradigm task in patients with schizophrenia," Schizophrenia Research, vol. 156, no. 2-3, pp. 197-203, 2014.

[36] E. van Diessen, T. Numan, E. van Dellen et al., "Opportunities and methodological challenges in EEG and MEG resting state functional brain network research," Clinical Neurophysiology, vol. 126, no. 8, pp. 1468-1481, 2015.

[37] J. Wang, X. Zuo, and Y. He, "Graph-based network analysis of resting-state functional MRI," Frontiers in Systems Neuroscience, vol. 4, article 16, 2010.

[38] E. T. Bullmore and D. S. Bassett, "Brain graphs: graphical models of the human brain connectome," Annual Review of Clinical Psychology, vol. 7, pp. 113-140, 2011.

[39] A. Fornito, A. Zalesky, and M. Breakspear, "Graph analysis of the human connectome: Promise, progress, and pitfalls," NeuroImage, vol. 80, pp. 426-444, 2013.

[40] J. A. Maldjian, E. M. Davenport, and C. T. Whitlow, "Graph theoretical analysis of resting-state MEG data: Identifying interhemispheric connectivity and the default mode," NeuroImage, vol. 96, pp. 88-94, 2014.

[41] M. Li, R. Zheng, H. Zhang, J. Wang, and Y. Pan, "Effective identification of essential proteins based on priori knowledge, network topology and gene expressions," Methods, vol. 67, no. 3, pp. 325-333, 2014.

[42] X. Peng, J. Wang, W. Peng, F. Wu, and Y. Pan, "Protein-protein interactions: detection, reliability assessment and applications," Briefings in Bioinformatics, vol. 18, no. 5, pp. 798-819, 2016.

[43] X. Peng, J. Wang, J. Huan, and F.-X. Wu, "Double-layer clustering method to predict protein complexes based on power-law distribution and protein sublocalization," Journal of Theoretical Biology, vol. 395, pp. 186-193, 2016.

[44] X. Peng, X. Yan, and J. Wang, "Framework to identify protein complexes based on similarity preclustering," Tsinghua Science and Technology, vol. 22, no. 1, pp. 42-51, 2017.

[45] O. Sporns, G. Tononi, and R. Kötter, “The human connectome: a structural description of the human brain," PLoS Computational Biology, vol. 1, no. 4, article e42, 2005.

[46] M. P. van den Heuvel, C. J. Stam, M. Boersma, and H. E. Hulshoff Pol, "Small-world and scale-free organization of voxelbased resting-state functional connectivity in the human brain," NeuroImage, vol. 43, no. 3, pp. 528-539, 2008.

[47] A. Fornito, A. Zalesky, C. Pantelis, and E. T. Bullmore, "Schizophrenia, neuroimaging and connectomics," NeuroImage, vol. 62, no. 4, pp. 2296-2314, 2012.
[48] M. Xia and Y. He, "Functional connectomics from a "big data" perspective," NeuroImage, 2016.

[49] P. Hagmann, L. Cammoun, X. Gigandet et al., "MR connectomics: Principles and challenges," Journal of Neuroscience Methods, vol. 194, no. 1, pp. 34-45, 2010.

[50] E. C. W. van Straaten and C. J. Stam, "Structure out of chaos: Functional brain network analysis with EEG, MEG, and functional MRI," European Neuropsychopharmacology, vol. 23, no. 1, pp. 7-18, 2013.

[51] E. Bullmore and O. Sporns, "Complex brain networks: graph theoretical analysis of structural and functional systems," Nature Reviews Neuroscience, vol. 10, no. 3, pp. 186-198, 2009.

[52] Q. K. Telesford, J. H. Burdette, and P. J. Laurienti, "An exploration of graph metric reproducibility in complex brain networks," Frontiers in Neuroscience, no. 7, article 67, 2013.

[53] F. Vecchio, F. Miraglia, D. Quaranta et al., "Cortical connectivity and memory performance in cognitive decline: A study via graph theory from EEG data," Neuroscience, vol. 316, pp. 143150, 2016.

[54] L. D. F. Costa, F. A. Rodrigues, G. Travieso, and P. R. V. Boas, "Characterization of complex networks: a survey of measurements," Advances in Physics, vol. 56, no. 1, pp. 167-242, 2007.

[55] A. Zalesky, L. Cocchi, A. Fornito, M. M. Murray, and E. Bullmore, "Connectivity differences in brain networks," Neurolmage, vol. 60, no. 2, pp. 1055-1062, 2012.

[56] D. Mears and H. B. Pollard, "Network science and the human brain: Using graph theory to understand the brain and one of its hubs, the amygdala, in health and disease," Journal of Neuroscience Research, vol. 94, no. 6, pp. 590-605, 2016.

[57] C. T. Butts, "Revisiting the foundations of network analysis," American Association for the Advancement of Science: Science, vol. 325, no. 5939, pp. 414-416, 2009.

[58] K. Brodmann, Vergleichende Lokalisationslehre der Grosshirnrinde in ihren Prinzipien dargestellt auf Grund des Zellenbaues, Barth, 1909.

[59] N. Tzourio-Mazoyer, B. Landeau, D. Papathanassiou et al., "Automated anatomical labeling of activations in SPM using a macroscopic anatomical parcellation of the MNI MRI singlesubject brain," NeuroImage, vol. 15, no. 1, pp. 273-289, 2002.

[60] D. B. Dwyer, B. J. Harrison, M. Yücel et al., "Large-scale brain network dynamics supporting adolescent cognitive control," The Journal of Neuroscience, vol. 34, no. 42, pp. 14096-14107, 2014.

[61] N. U. F. Dosenbach, B. Nardos, A. L. Cohen et al., "Prediction of individual brain maturity using fMRI," Science, vol. 329, no. 5997, pp. 1358-1361, 2010.

[62] C. Reveley, A. K. Seth, C. Pierpaoli et al., "Superficial white matter fiber systems impede detection of long-range cortical connections in diffusion MR tractography," Proceedings of the National Acadamy of Sciences of the United States of America, vol. 112, no. 21, pp. E2820-E2828, 2015.

[63] R. E. Passingham, K. E. Stephan, and R. Kötter, “The anatomical basis of functional localization in the cortex," Nature Reviews Neuroscience, vol. 3, no. 8, pp. 606-616, 2002.

[64] A. Anwander, M. Tittgemeyer, D. Y. Von Cramon, A. D. Friederici, and T. R. Knösche, "Connectivity-based parcellation of Broca's area," Cerebral Cortex, vol. 17, no. 4, pp. 816-825, 2007.

[65] J. D. Power, A. L. Cohen, S. M. Nelson et al., "Functional network organization of the human brain," Neuron, vol. 72, no. 4 , pp. 665-678, 2011. 
[66] B. T. T. Yeo, F. M. Krienen, J. Sepulcre et al., “The organization of the human cerebral cortex estimated by intrinsic functional connectivity," Journal of Neurophysiology, vol. 106, no. 3, pp. 1125-1165, 2011.

[67] R. C. Craddock, G. A. James, P. E. Holtzheimer, X. P. Hu, and H. S. Mayberg, "A whole brain fMRI atlas generated via spatially constrained spectral clustering," Human Brain Mapping, vol. 33, no. 8, pp. 1914-1928, 2012.

[68] D. Moreno-Dominguez, A. Anwander, and T. R. Knösche, "A hierarchical method for whole-brain connectivity-based parcellation," Human Brain Mapping, vol. 35, no. 10, pp. 5000-5025, 2014.

[69] M. F. Glasser, T. S. Coalson, E. C. Robinson et al., "A multimodal parcellation of human cerebral cortex," Nature, vol. 536, no. 7615, pp. 171-178, 2016.

[70] K. J. Friston, "Functional and effective connectivity: a review," Brain Connectivity, vol. 1, no. 1, pp. 13-36, 2011.

[71] D. S. Bassett, E. Bullmore, B. A. Verchinski, V. S. Mattay, D. R. Weinberger, and A. Meyer-Lindenberg, "Hierarchical organization of human cortical networks in health and Schizophrenia," The Journal of Neuroscience, vol. 28, no. 37, pp. 9239-9248, 2008.

[72] Y. Zhang, L. Lin, C.-P. Lin et al., "Abnormal topological organization of structural brain networks in schizophrenia," Schizophrenia Research, vol. 141, no. 2-3, pp. 109-118, 2012.

[73] A. Alexander-Bloch, J. N. Giedd, and E. Bullmore, "Imaging structural co-variance between human brain regions," Nature Reviews Neuroscience, vol. 14, no. 5, pp. 322-336, 2013.

[74] D. S. Tuch, T. G. Reese, M. R. Wiegell, and V. J. Wedeen, "Diffusion MRI of complex neural architecture," Neuron, vol. 40, no. 5, pp. 885-895, 2003.

[75] D. S. Tuch, "Q-ball imaging," Magnetic Resonance in Medicine, vol. 52, no. 6, pp. 1358-1372, 2004.

[76] V. J. Wedeen, P. Hagmann, W.-Y. I. Tseng, T. G. Reese, and R. M. Weisskoff, "Mapping complex tissue architecture with diffusion spectrum magnetic resonance imaging," Magnetic Resonance in Medicine, vol. 54, no. 6, pp. 1377-1386, 2005.

[77] S. Mori and J. Zhang, "Principles of diffusion tensor imaging and its applications to basic neuroscience research," Neuron, vol. 51, no. 5, pp. 527-539, 2006.

[78] T. E. Conturo, N. F. Lori, T. S. Cull et al., "Tracking neuronal fiber pathways in the living human brain," Proceedings of the National Acadamy of Sciences of the United States of America, vol. 96, no. 18, pp. 10422-10427, 1999.

[79] P. J. Basser, S. Pajevic, C. Pierpaoli, J. Duda, and A. Aldroubi, "In vivo fiber tractography using DT-MRI data," Magnetic Resonance in Medicine, vol. 44, no. 4, pp. 625-632, 2000.

[80] T. E. J. Behrens, H. J. Berg, S. Jbabdi, M. F. S. Rushworth, and M. W. Woolrich, "Probabilistic diffusion tractography with multiple fibre orientations: what can we gain?" NeuroImage, vol. 34, no. 1, pp. 144-155, 2007.

[81] J.-D. Tournier, S. Mori, and A. Leemans, "Diffusion tensor imaging and beyond," Magnetic Resonance in Medicine, vol. 65, no. 6, pp. 1532-1556, 2011.

[82] S. Jbabdi and H. Johansen-Berg, "Tractography: where do we go from here?” Brain Connectivity, vol. 1, no. 3, pp. 169-183, 2011.

[83] Z. Yao, Y. Zhang, L. Lin et al., "Abnormal cortical networks in mild cognitive impairment and Alzheimer's disease," PLoS Computational Biology, vol. 6, no. 11, Article ID e1001006, 2010.

[84] N. Shu, Y. Liang, H. Li et al., "Disrupted topological organization in white matter structural networks in amnestic mild cognitive impairment: relationship to subtype," Radiology, vol. 265, no. 2, pp. 518-527, 2012.
[85] M. R. Brier, J. B. Thomas, A. M. Fagan et al., "Functional connectivity and graph theory in preclinical Alzheimer's disease," Neurobiology of Aging, vol. 35, no. 4, pp. 757-768, 2014.

[86] S. Golbabaei, A. Dadashi, and H. Soltanian-Zadeh, "Measures of the brain functional network that correlate with Alzheimer's neuropsychological test scores: An fMRI and graph analysis study," in Proceedings of the 38th Annual International Conference of the IEEE Engineering in Medicine and Biology Society, EMBC 2016, pp. 5554-5557, August 2016.

[87] Z. Yin, J. Li, Y. Zhang, A. Ren, K. M. Von Meneen, and L. Huang, "Functional brain network analysis of schizophrenic patients with positive and negative syndrome based on mutual information of EEG time series," Biomedical Signal Processing and Control, vol. 31, pp. 331-338, 2017.

[88] K. Supekar, V. Menon, D. Rubin, M. Musen, and M. D. Greicius, "Network analysis of intrinsic functional brain connectivity in Alzheimer's disease," PLoS Computational Biology, vol. 4, no. 6, Article ID e1000100, 2008.

[89] J. Wang, X. Zuo, Z. Dai et al., "Disrupted functional brain connectome in individuals at risk for Alzheimer's disease," Biological Psychiatry, vol. 73, no. 5, pp. 472-481, 2013.

[90] B. Fischl, A. van der Kouwe, C. Destrieux et al., "Automatically parcellating the human cerebral cortex," Cerebral Cortex, vol. 14, no. 1, pp. 11-22, 2004.

[91] C. Destrieux, B. Fischl, A. Dale, and E. Halgren, "Automatic parcellation of human cortical gyri and sulci using standard anatomical nomenclature," NeuroImage, vol. 53, no. 1, pp. 1-15, 2010.

[92] R. S. Desikan, F. Ségonne, B. Fischl et al., "An automated labeling system for subdividing the human cerebral cortex on MRI scans into gyral based regions of interest," NeuroImage, vol. 31, no. 3, pp. 968-980, 2006.

[93] D. W. Shattuck, M. Mirza, V. Adisetiyo et al., "Construction of a 3D probabilistic atlas of human cortical structures," NeuroImage, vol. 39, no. 3, pp. 1064-1080, 2008.

[94] L. Fan, H. Li, J. Zhuo et al., "The human brainnetome atlas: a new brain atlas based on connectional architecture," Cerebral Cortex, vol. 26, no. 8, pp. 3508-3526, 2016.

[95] Y. He, Z. Chen, and A. Evans, "Structural insights into aberrant topological patterns of large-scale cortical networks in Alzheimer's disease," The Journal of Neuroscience, vol. 28, no. 18, pp. 4756-4766, 2008.

[96] B. M. Tijms, C. Möller, H. Vrenken et al., "Single-subject grey matter graphs in Alzheimer's disease," PLoS ONE, vol. 8, no. 3, Article ID e58921, 2013.

[97] J. B. Pereira, M. Mijalkov, E. Kakaei et al., "Disrupted network topology in patients with stable and progressive mild cognitive impairment and Alzheimer's disease," Cerebral Cortex, vol. 26, no. 8, pp. 3476-3493, 2016.

[98] C.-Y. Lo, P.-N. Wang, K.-H. Chou, J. Wang, Y. He, and C.-P. Lin, "Diffusion tensor tractography reveals abnormal topological organization in structural cortical networks in Alzheimer's disease," The Journal of Neuroscience, vol. 30, no. 50, pp. 1687616885, 2010.

[99] F. Bai, N. Shu, Y. Yuan et al., “Topologically convergent and divergent structural connectivity patterns between patients with remitted geriatric depression and amnestic mild cognitive impairment," The Journal of Neuroscience, vol. 32, no. 12, pp. 4307-4318, 2012.

[100] M. Daianu, N. Jahanshad, T. M. Nir et al., "Rich club analysis in the Alzheimer's disease connectome reveals a relatively 
undisturbed structural core network," Human Brain Mapping, vol. 36, no. 8, pp. 3087-3103, 2015.

[101] T. Wang, F. Shi, Y. Jin et al., "Multilevel deficiency of white matter connectivity networks in Alzheimer's disease: a diffusion MRI study with DTI and HARDI models," Neural Plasticity, vol. 2016, Article ID 2947136, 14 pages, 2016.

[102] A. Zalesky, A. Fornito, I. H. Harding et al., "Whole-brain anatomical networks: does the choice of nodes matter?" NeuroImage, vol. 50, no. 3, pp. 970-983, 2010.

[103] M. M. A. Engels, C. J. Stam, W. M. van der Flier, P. Scheltens, H. de Waal, and E. C. W. van Straaten, "Declining functional connectivity and changing hub locations in Alzheimer's disease: An EEG study," BMC Neurology, vol. 15, no. 1, article no. 145, 2015.

[104] Y. Deng, L. Shi, Y. Lei, and D. Wang, "Altered topological organization of high-level visual networks in Alzheimer's disease and mild cognitive impairment patients," Neuroscience Letters, vol. 630, pp. 147-153, 2016.

[105] F. Shi, P.-T. Yap, W. Gao, W. Lin, J. H. Gilmore, and D. Shen, "Altered structural connectivity in neonates at genetic risk for schizophrenia: a combined study using morphological and white matter networks," NeuroImage, vol. 62, no. 3, pp. 16221633, 2012.

[106] B. M. Tijms, E. Sprooten, D. Job et al., "Grey matter networks in people at increased familial risk for schizophrenia," Schizophrenia Research, vol. 168, no. 1-2, article no. 6513, pp. 1-8, 2015.

[107] A. Zalesky, A. Fornito, M. L. Seal et al., "Disrupted axonal fiber connectivity in schizophrenia," Biological Psychiatry, vol. 69, no. 1, pp. 80-89, 2011.

[108] G. Collin, R. S. Kahn, M. A. De Reus, W. Cahn, and M. P. Van Den Heuvel, "Impaired rich club connectivity in unaffected siblings of schizophrenia patients," Schizophrenia Bulletin, vol. 40, no. 2, pp. 438-448, 2014.

[109] Y. Sun, Y. Chen, S. L. Collinson, A. Bezerianos, and K. Sim, "Reduced hemispheric asymmetry of brain anatomical networks is linked to schizophrenia: A connectomics study," Cerebral Cortex, vol. 1, article 14, 2015.

[110] Y. Sun, Y. Chen, R. Lee, A. Bezerianos, S. L. Collinson, and K. Sim, "Disruption of brain anatomical networks in schizophrenia: A longitudinal, diffusion tensor imaging based study," Schizophrenia Research, vol. 171, no. 1-3, pp. 149-157, 2016.

[111] M.-E. Lynall, D. S. Bassett, R. Kerwin et al., "Functional connectivity and brain networks in schizophrenia," The Journal of Neuroscience, vol. 30, no. 28, pp. 9477-9487, 2010.

[112] T.-W. Su, T.-W. Hsu, Y.-C. Lin, and C.-P. Lin, "Schizophrenia symptoms and brain network efficiency: A resting-state fMRI study," Psychiatry Research: Neuroimaging, vol. 234, no. 2, pp. 208-218, 2015.

[113] J. A. Hadley, N. V. Kraguljac, D. M. White, L. Ver Hoef, J. Tabora, and A. C. Lahti, "Change in brain network topology as a function of treatment response in schizophrenia: a longitudinal resting-state fMRI study using graph theory," Schizophrenia, vol. 2, no. 1, article 16014, 2016.

[114] X. Shen, F. Tokoglu, X. Papademetris, and R. T. Constable, "Groupwise whole-brain parcellation from resting-state fMRI data for network node identification," NeuroImage, vol. 82, pp. 403-415, 2013.

[115] E. P. Ganella, C. F. Bartholomeusz, C. Seguin et al., "Functional brain networks in treatment-resistant schizophrenia," Schizophrenia Research, vol. 184, pp. 73-81, 2017.
[116] K. Jhung, S.-H. Cho, J.-H. Jang et al., "Small-world networks in individuals at ultra-high risk for psychosis and first-episode schizophrenia during a working memory task," Neuroscience Letters, vol. 535, no. 1, pp. 35-39, 2013.

[117] D. Zhang, J. Wang, X. Liu, J. Chen, and B. Liu, "Aberrant brain network efficiency in Parkinson's disease patients with tremor: A multi-modality study," Frontiers in Aging Neuroscience, vol. 7, article no. 169, 2015.

[118] J. B. Pereira, D. Aarsland, C. E. Ginestet et al., "Aberrant cerebral network topology and mild cognitive impairment in early Parkinson's disease," Human Brain Mapping, vol. 36, no. 8, pp. 2980-2995, 2015.

[119] S. K. Yadav, N. Kathiresan, S. Mohan et al., "Gender-based analysis of cortical thickness and structural connectivity in Parkinson's disease," Journal of Neurology, vol. 263, no. 11, pp. 2308-2318, 2016.

[120] C. Li, B. Huang, R. Zhang et al., "Impaired topological architecture of brain structural networks in idiopathic Parkinson's disease: a DTI study," Brain Imaging and Behavior, vol. 11, no. 1, pp. 113-128, 2017.

[121] S. Nigro, R. Riccelli, L. Passamonti et al., "Characterizing structural neural networks in de novo Parkinson disease patients using diffusion tensor imaging," Human Brain Mapping, vol. 37, no. 12, pp. 4500-4510, 2016.

[122] F. Skidmore, D. Korenkevych, Y. Liu, G. He, E. Bullmore, and P. M. Pardalos, "Connectivity brain networks based on wavelet correlation analysis in Parkinson fMRI data," Neuroscience Letters, vol. 499, no. 1, pp. 47-51, 2011.

[123] M. Göttlich, T. F. Münte, M. Heldmann, M. Kasten, J. Hagenah, and U. M. Krämer, "Altered resting state brain networks in Parkinson's disease," PLoS ONE, vol. 8, no. 10, Article ID e77336, 2013.

[124] C. Y. Luo, X. Y. Guo, W. Song et al., "Functional connectome assessed using graph theory in drug-naive Parkinson's disease," Journal of Neurology, vol. 262, no. 6, pp. 1557-1567, 2015.

[125] Y. Koshimori, S.-S. Cho, M. Criaud et al., "Disrupted nodal and hub organization account for brain network abnormalities in Parkinson's disease," Frontiers in Aging Neuroscience, vol. 8, article no. 259, 2016.

[126] K. T. E. Olde Dubbelink, A. Hillebrand, D. Stoffers et al., "Disrupted brain network topology in Parkinson's disease: a longitudinal magnetoencephalography study," Brain, vol. 137, no. 1, pp. 197-207, 2014.

[127] R. L. Utianski, J. N. Caviness, E. C. W. van Straaten et al., "Graph theory network function in parkinson's disease assessed with electroencephalography," Clinical Neurophysiology, vol. 127, no. 5, pp. 2228-2236, 2016.

[128] Y. He, A. Dagher, Z. Chen et al., "Impaired small-world efficiency in structural cortical networks in multiple sclerosis associated with white matter lesion load," Brain, vol. 132, no. 12, pp. 3366-3379, 2009.

[129] P. Tewarie, M. D. Steenwijk, B. M. Tijms et al., "Disruption of structural and functional networks in long-standing multiple sclerosis," Human Brain Mapping, vol. 35, no. 12, pp. 5946-5961, 2014.

[130] N. Shu, Y. Liu, K. Li et al., "Diffusion tensor tractography reveals disrupted topological efficiency in white matter structural networks in multiple sclerosis," Cerebral Cortex, vol. 21, no. 11, pp. 2565-2577, 2011.

[131] S. Nigro, L. Passamonti, R. Riccelli et al., "Structural 'connectomic' alterations in the limbic system of multiple sclerosis 
patients with major depression," Multiple Sclerosis Journal, vol. 21, no. 8, pp. 1003-1012, 2015.

[132] N. Shu, Y. Duan, M. Xia et al., "Disrupted topological organization of structural and functional brain connectomes in clinically isolated syndrome and multiple sclerosis," Scientific Reports, vol. 6, Article ID 29383, 2016.

[133] S. Llufriu, E. Martinez-Heras, E. Solana et al., "Structural networks involved in attention and executive functions in multiple sclerosis," NeuroImage: Clinical, vol. 13, pp. 288-296, 2017.

[134] O. L. Gamboa, E. Tagliazucchi, F. von Wegner et al., "Working memory performance of early MS patients correlates inversely with modularity increases in resting state functional connectivity networks," NeuroImage, vol. 94, pp. 385-395, 2014.

[135] M. A. Rocca, P. Valsasina, A. Meani, A. Falini, G. Comi, and M. Filippi, "Impaired functional integration in multiple sclerosis: a graph theory study," Brain Structure and Function, vol. 221, no. 1, pp. 115-131, 2016.

[136] Y. Liu, H. Wang, Y. Duan et al., "Functional brain network alterations in clinically isolated syndrome and multiple sclerosis: A graph-based connectome study," Radiology, vol. 282, no. 2, pp. 534-541, 2017.

[137] M. M. Schoonheim, J. J. G. Geurts, D. Landi et al., "Functional connectivity changes in multiple sclerosis patients: A graph analytical study of MEG resting state data," Human Brain Mapping, vol. 34, no. 1, pp. 52-61, 2013.

[138] N. A. Crossley, A. Mechelli, J. Scott et al., "The hubs of the human connectome are generally implicated in the anatomy of brain disorders," Brain, vol. 137, no. 8, pp. 2382-2395, 2015.

[139] M. P. van den Heuvel and O. Sporns, "Network hubs in the human brain," Trends in Cognitive Sciences, vol. 17, no. 12, pp. 683-696, 2013.

[140] M. Rubinov and O. Sporns, "Complex network measures of brain connectivity: Uses and interpretations," NeuroImage, vol. 52, no. 3, pp. 1059-1069, 2010.

[141] M. A. Beauchamp, "An improved index of centrality," Behavioural Science, vol. 10, pp. 161-163, 1965.

[142] J. M. Anthonisse, “The rush in a directed graph," Stichting Mathematisch Centrum. Mathematische Besliskunde, no. BN 9/71, pp. 1-10, 1971.

[143] L. C. Freeman, "A set of measures of centrality based on betweenness," Sociometry, vol. 40, no. 1, pp. 35-41, 1977.

[144] S. Achard and E. Bullmore, "Efficiency and cost of economical brain functional networks," PLoS Computational Biology, vol. 3, no. 2, p. e17, 2007.

[145] V. Latora and M. Marchiori, "Efficient behavior of small-world networks," Physical Review Letters, vol. 87, no. 19, Article ID 198701, 2001.

[146] O. Sporns, C. J. Honey, and R. Kötter, "Identification and classification of hubs in brain networks," PLOS ONE, vol. 2, no. 10, Article ID e1049, 2007.

[147] M. E. J. Newman and M. Girvan, "Finding and evaluating community structure in networks," Physical Review E: Statistical, Nonlinear, and Soft Matter Physics, vol. 69, no. 2, Article ID 026113, pp. 1-26113, 2004.

[148] A. Fornito, A. Zalesky, and E. Bullmore, Fundamentals of Brain Network Analysis, Academic Press, 2016.

[149] S. Zhou and R. J. Mondragón, "The rich-club phenomenon in the internet topology," IEEE Communications Letters, vol. 8, no. 3, pp. 180-182, 2004.
[150] V. Colizza, A. Flammini, M. A. Serrano, and A. Vespignani, "Detecting rich-club ordering in complex networks," Nature Physics, vol. 2, no. 2, pp. 110-115, 2006.

[151] M. Kocher, E. Gleichgerrcht, T. Nesland et al., "Individual variability in the anatomical distribution of nodes participating in rich club structural networks," Frontiers in Neural Circuits, vol. 9, article no. 16, 2015.

[152] M. P. van den Heuvel and O. Sporns, "Rich-club organization of the human connectome," The Journal of Neuroscience, vol. 31, no. 44, pp. 15775-15786, 2011.

[153] M. Senden, G. Deco, M. A. De Reus, R. Goebel, and M. P. Van Den Heuvel, "Rich club organization supports a diverse set of functional network configurations," NeuroImage, vol. 96, pp. 174-182, 2014.

[154] D. J. Watts and S. H. Strogatz, "Collective dynamics of "smallworld” networks," Nature, vol. 393, no. 6684, pp. 440-442, 1998.

[155] M. D. Humphries, K. Gurney, and T. J. Prescott, "The brainstem reticular formation is a small-world, not scale-free, network," Proceedings of the Royal Society of London B: Biological, vol. 273, no. 1585, pp. 503-511, 2006.

[156] S. Boccaletti, V. Latora, Y. Moreno, M. Chavez, and D.-U. Hwang, "Complex networks: structure and dynamics," Physics Reports, vol. 424, no. 4-5, pp. 175-308, 2006.

[157] A. Association, "2014 Alzheimers Disease Facts and Figures," Alzheimers \& Dementia, vol. 10, no. 2, pp. e47-e92, 2014.

[158] R. C. Petersen, "Mild cognitive impairment as a diagnostic entity," Journal of Internal Medicine, vol. 256, no. 3, pp. 183-194, 2004.

[159] B. M. Tijms, A. M. Wink, W. de Haan et al., "Alzheimer's disease: connecting findings from graph theoretical studies of brain networks," Neurobiology of Aging, vol. 34, no. 8, pp. 2023-2036, 2013.

[160] M. P. Van Den Heuvel and A. Fornito, "Brain networks in schizophrenia," Neuropsychology Review, vol. 24, no. 1, pp. 3248, 2014.

[161] A. L. Wheeler and A. N. Voineskos, "A review of structural neuroimaging in schizophrenia: From connectivity to connectomics," Frontiers in Human Neuroscience, vol. 8, article no. 653, 2014.

[162] R. Zhang, Q. Wei, Z. Kang et al., "Disrupted brain anatomical connectivity in medication-naïve patients with first-episode schizophrenia," Brain structure \& function, vol. 220, no. 2, pp. 1145-1159, 2015.

[163] A. W. Willis, "Parkinson disease in the elderly adult.," Missouri Medicine, vol. 110, no. 5, pp. 406-410, 2013.

[164] C. P. Weingarten, M. H. Sundman, P. Hickey, and N.-K. Chen, "Neuroimaging of Parkinson's disease: Expanding views," Neuroscience \& Biobehavioral Reviews, vol. 59, pp. 16-52, 2015.

[165] Q. Cao, N. Shu, L. An et al., "Probabilistic diffusion tractography and graph theory analysis reveal abnormal white matter structural connectivity networks in drug-naive boys with attention deficit/hyperactivity disorder," The Journal of Neuroscience, vol. 33, no. 26, pp. 10676-10687, 2013.

[166] G. Alba, E. Pereda, S. Mañas et al., “The variability of EEG functional connectivity of young ADHD subjects in different resting states," Clinical Neurophysiology, vol. 127, no. 2, pp. 13211330, 2016.

[167] R. Beare, C. Adamson, M. A. Bellgrove et al., "Altered structural connectivity in ADHD: a network based analysis," Brain Imaging and Behavior, vol. 11, no. 3, pp. 846-858, 2017. 
[168] E. Van Diessen, W. J. E. M. Zweiphenning, F. E. Jansen, C. J. Stam, K. P. J. Braun, and W. M. Otte, "Brain network organization in focal epilepsy: A systematic review and metaanalysis," PLoS ONE, vol. 9, no. 12, Article ID el14606, 2014.

[169] B. C. Bernhardt, L. Bonilha, and D. W. Gross, "Network analysis for a network disorder: The emerging role of graph theory in the study of epilepsy," Epilepsy \& Behavior, vol. 50, pp. 162-170, 2015.

[170] J. Wirsich, A. Perry, B. Ridley et al., "Whole-brain analytic measures of network communication reveal increased structurefunction correlation in right temporal lobe epilepsy," NeuroImage: Clinical, vol. 11, pp. 707-718, 2016.

[171] J. D. Rudie, J. A. Brown, D. Beck-Pancer et al., "Altered functional and structural brain network organization in autism," NeuroImage: Clinical, vol. 2, no. 1, pp. 79-94, 2013.

[172] L. M. Hernandez, J. D. Rudie, S. A. Green, S. Bookheimer, and M. Dapretto, "Neural signatures of autism spectrum disorders: Insights into brain network dynamics," Neuropsychopharmacology, vol. 40, no. 1, pp. 171-189, 2015.

[173] J. D. Lewis, A. C. Evans, J. R. Pruett et al., "The Emergence of Network Inefficiencies in Infants With Autism Spectrum Disorder," Biological Psychiatry, vol. 82, no. 3, pp. 176-185, 2017.

[174] H.-J. Park and K. Friston, "Structural and functional brain networks: from connections to cognition," Science, vol. 342, no. 6158, Article ID 1238411, 2013.

[175] A. Messé, D. Rudrauf, H. Benali, and G. Marrelec, "Relating Structure and Function in the Human Brain: Relative Contributions of Anatomy, Stationary Dynamics, and Nonstationarities," PLoS Computational Biology, vol. 10, no. 3, Article ID e1003530, 2014.

[176] M. De Domenico, S. Sasai, and A. Arenas, "Mapping multiplex hubs in human functional brain networks," Frontiers in Neuroscience, vol. 10, article no. 326, 2016.

[177] F. Battiston, V. Nicosia, M. Chavez, and V. Latora, "Multilayer motif analysis of brain networks," Chaos: An Interdisciplinary Journal of Nonlinear Science, vol. 27, no. 4, article 047404, 2017.

[178] M. De Domenico, "Multilayer modeling and analysis of human brain networks," Giga Science, vol. 6, no. 5, pp. 1-8, 2017. 


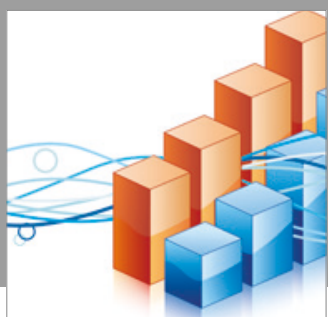

Advances in

Operations Research

vatersals

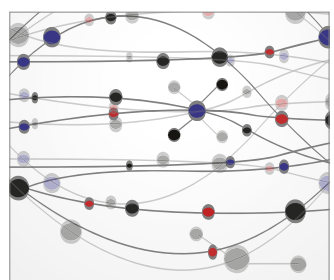

\section{The Scientific} World Journal
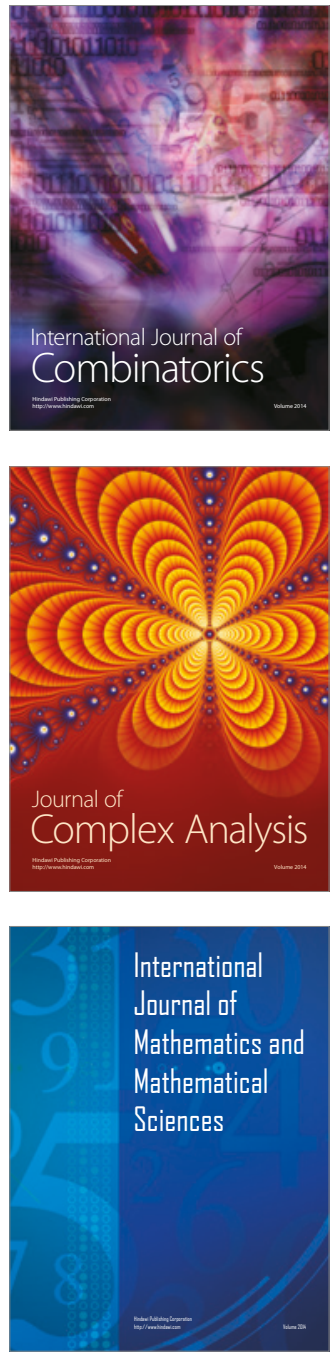
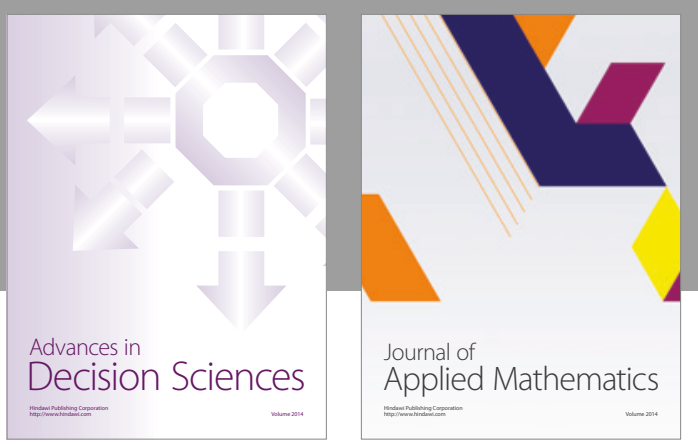

Algebra

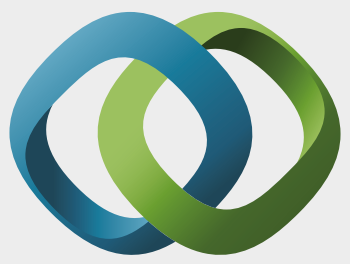

\section{Hindawi}

Submit your manuscripts at

https://www.hindawi.com
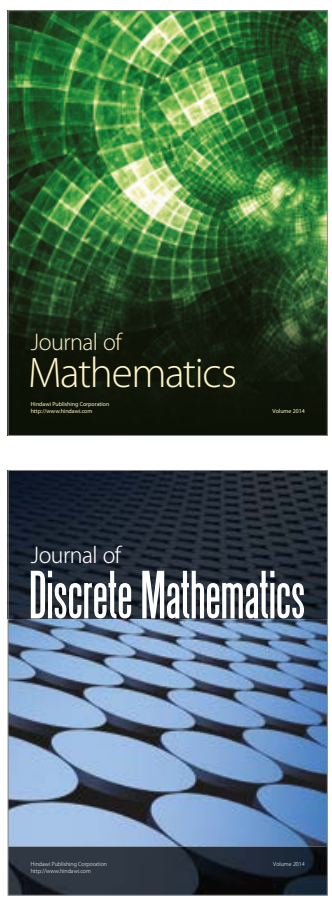

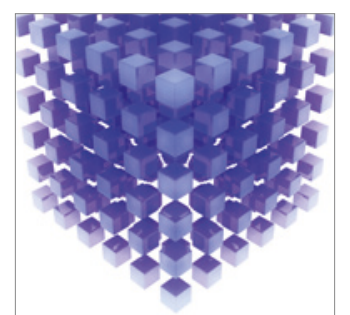

Mathematical Problems in Engineering
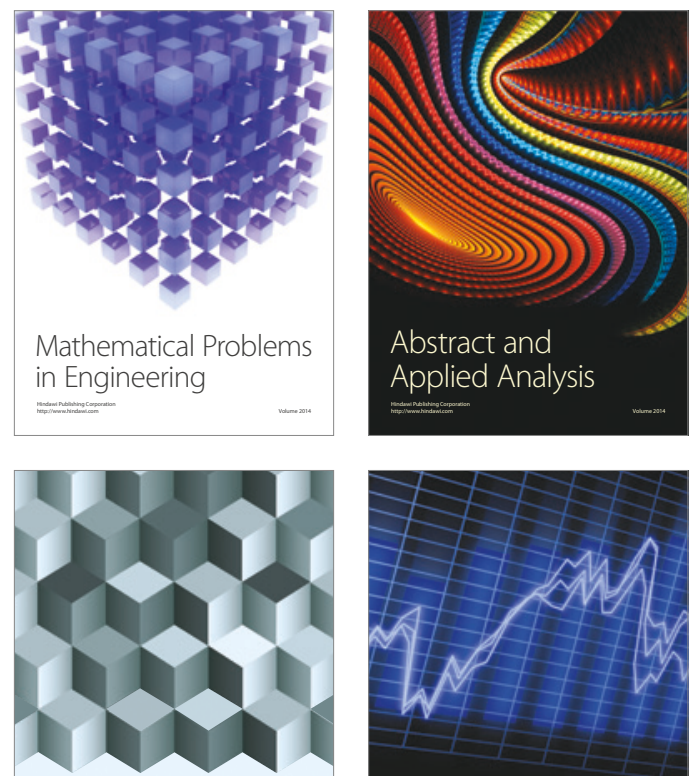

Journal of

Function Spaces

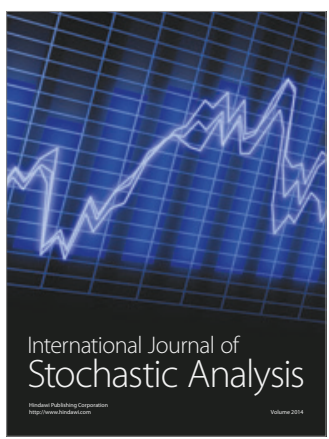

Probability and Statistics
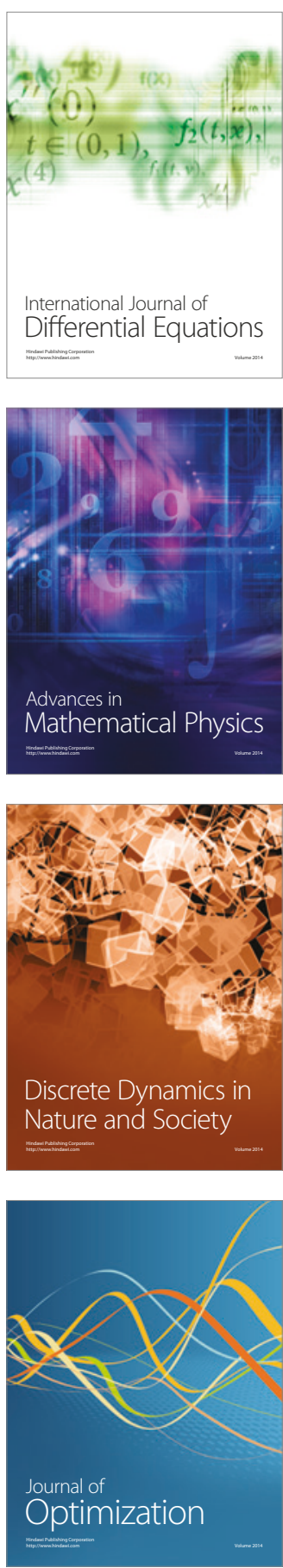